\title{
Error analysis for the approximation of axisymmetric Willmore flow by $C^{1}$-finite elements
}

\author{
KLAUS DECKELNICK AND FRIEDHELM SCHIEWECK \\ Institut für Analysis und Numerik, Otto-von-Guericke-Universität Magdeburg, \\ Universitätsplatz 2, 39106 Magdeburg, Germany \\ E-mail: klaus.deckelnick@ovgu.de, schiewec@ovgu.de
}

[Received 14 September 2009 and in revised form 30 July 2010]

\begin{abstract}
We consider the Willmore flow of axially symmetric surfaces subject to Dirichlet boundary conditions. The corresponding evolution is described by a nonlinear parabolic PDE of fourth order for the radius function. A suitable weak form of the equation, which is based on the first variation of the Willmore energy, leads to a semidiscrete scheme, in which we employ piecewise cubic $C^{1}$-finite elements for the one-dimensional approximation in space. We prove optimal error bounds in Sobolev norms for the solution and its time derivative and present numerical test examples.
\end{abstract}

2010 Mathematics Subject Classification: 35K60, 65M15, 65M60.

Keywords: Willmore flow; Dirichlet boundary conditions; finite elements; error estimates.

\section{Introduction}

Let $\Gamma$ be a smooth, oriented hypersurface in $\mathbb{R}^{3}$. The Willmore functional is defined by

$$
W(\Gamma)=\int_{\Gamma} H^{2} \mathrm{~d} A
$$

where $H=\frac{1}{2}\left(\kappa_{1}+\kappa_{2}\right)$ denotes the mean curvature of $\Gamma$ and $\kappa_{1}, \kappa_{2}$ are the principal curvatures. We use the convention that a sphere has positive mean curvature if the orientation is given by the unit outer normal. In view of its invariance with respect to conformal transformations of $\mathbb{R}^{3}$, the Willmore functional has been intensively studied in differential geometry (see [22] for an introduction). The functional $W$ and suitable generalizations also serve as mathematical models for the bending energy of thin plates and biological cell membranes (see e.g. [12], [20]). In this paper we focus on the $L^{2}$-gradient flow of $W$, the so-called Willmore flow, which is given by the following geometric evolution equation:

$$
V=\Delta_{\Gamma} H+2 H^{3}-2 H K \quad \text { on } \Gamma(t) .
$$

Here, $V$ denotes the normal velocity of $(\Gamma(t))_{t \in[0, T]}, K=\kappa_{1} \kappa_{2}$ is the Gauss curvature and $\Delta_{\Gamma}$ is the Laplace-Beltrami operator. Depending on the representation of the evolving surfaces, (1.2) leads to a nonlinear parabolic equation or system which is of fourth order in space. Global existence results for the evolution of closed surfaces can be found in [14], [15]. In addition, two boundary conditions need to be prescribed in case the evolving surfaces have a boundary. Willmore flow coupled with boundary conditions has been used in image processing for problems related to surface restoration and image inpainting (see [4] and the references therein). Furthermore, one can use [1.2] in order 
to calculate local or global minima of $W$ by considering the limits $t \rightarrow \infty$. A rigorous proof of the existence of such minima subject to Dirichlet boundary conditions has been obtained in the axially symmetric case in [5], [6], while the parametric case is investigated in [19].

This paper is concerned with the numerical approximation of axially symmetric solutions of (1.2) subject to Dirichlet boundary conditions. Before we describe our setting let us briefly review the literature on numerical methods for Willmore flow. In [13], a discrete version of (1.1) was minimized with the help of Brakke's surface evolver ([3]). Mayer and Simonett ([16]) use a finite difference scheme in order to approximate axisymmetric solutions of Willmore flow. Finite element discretizations for parametric Willmore flow are presented in [18], [1], [10] and [17]. These methods have in common that they split the fourth order problem into two second order problems for the position and either the scalar mean curvature or the mean curvature vector. These variables are then approximated by piecewise linear finite elements. A discretization based on quadratic isoparametric elements is introduced in [2]. While the above-mentioned papers treat closed surfaces, the evolution of surfaces with boundaries has been less investigated. In [9], a level set approach to Willmore flow is presented into which boundary conditions can be incorporated. Again a splitting technique is applied using the level set function and a weighted mean curvature as variables. In view of its formal similarity, this approach can also be employed to discretize the Willmore flow of graphs. [7] contains an error analysis for the Willmore flow of graphs that evolve subject to boundary conditions in which the height of the graph is given and its mean curvature vanishes. In [4], the parametric approach from [18] is extended to surfaces with boundaries and applied to problems in surface restoration.

In this paper we shall focus on surfaces of revolution of the form

$$
\Gamma(t)=\left\{\mathbf{x} \in \mathbb{R}^{3} \mid \mathbf{x}=(x, u(x, t) \cos \varphi, u(x, t) \sin \varphi), x \in \bar{I}, \varphi \in[0,2 \pi]\right\},
$$

evolving by 1.2 . Here, $I=(-1,1)$ and $u: \bar{I} \times[0, T] \rightarrow \mathbb{R}$ is smooth and positive. The evolution law (1.2) translates into a nonlinear parabolic PDE of fourth order for the radius function $u$. We impose Dirichlet boundary conditions, i.e. $u( \pm 1, t)$ and $u_{x}( \pm 1, t)$ are prescribed for $t \in[0, T]$. The corresponding initial-boundary value problem along with a variational formulation involving test functions in $H_{0}^{2}(I)$ is derived in Section 2. In Section 3 we use that weak form in order to discretize in space. We employ a conforming finite element method using piecewise cubic polynomials which are globally $C^{1}$. This leads to an approximation of order 4 in the $L^{2}$-norm, which allows us to work with relatively coarse meshes and avoids the introduction of a second variable required for the splitting approach mentioned above. Our main result are the following error estimates for the semidiscrete method:

$$
\left(\int_{0}^{T}\left\|u_{t}-u_{h t}\right\|^{2} \mathrm{~d} t\right)^{1 / 2}+\sum_{i=0}^{2} h^{i} \max _{0 \leqslant t \leqslant T}\left\|\partial_{x}^{i}\left(u-u_{h}\right)(\cdot, t)\right\| \leqslant C h^{4} .
$$

Here, $\|\cdot\|$ denotes the $L^{2}$-norm. In Section 4 we introduce a suitable nonlinear projection that is adapted to the elliptic part of the underlying operator and which plays a key role in the analysis. The proof of the above bounds is presented in Section 5. In order to obtain a practical algorithm one still needs to discretize in time. We will employ the Crank-Nicolson scheme, which is of order 2, allowing us to take better advantage of the high approximation order in space. Details along with corresponding numerical tests can be found in Section 6. 


\section{Variational formulation}

The aim of this section is to derive a variational formulation for the evolution of surfaces of revolution under Willmore flow. To begin, consider

$$
\Gamma=\left\{\mathbf{x} \in \mathbb{R}^{3} \mid \mathbf{x}=(x, v(x) \cos \varphi, v(x) \sin \varphi), x \in \bar{I}, \varphi \in[0,2 \pi]\right\},
$$

where the function $v$ belongs to the set

$$
M:=\left\{v \in H^{2}(I) \mid v>0 \text { in } I, v(-1)=\alpha_{l}, v(1)=\alpha_{r}, v^{\prime}(-1)=\beta_{l}, v^{\prime}(1)=\beta_{r}\right\} .
$$

Here $\alpha_{l}, \alpha_{r}, \beta_{l}, \beta_{r}$ are given real numbers and $\alpha_{l}, \alpha_{r}>0$. Note that the definition of $M$ takes into account the fact that we intend to prescribe clamped boundary conditions. A straightforward calculation shows that the first and second fundamental forms of the above surface are given by

$$
\left(g_{i j}(x, \varphi)\right)=\left(\begin{array}{cc}
1+v^{\prime}(x)^{2} & 0 \\
0 & v(x)^{2}
\end{array}\right), \quad\left(h_{i j}(x, \varphi)\right)=\frac{1}{\sqrt{1+v^{\prime}(x)^{2}}}\left(\begin{array}{cc}
-v^{\prime \prime}(x) & 0 \\
0 & v(x)
\end{array}\right),
$$

where the orientation is the one induced by

$$
v(x, \varphi)=\frac{1}{\sqrt{1+v^{\prime}(x)^{2}}}\left(-v^{\prime}(x), \cos \varphi, \sin \varphi\right)
$$

The inverse matrix $\left(g^{i j}\right)$ and the area element are given by

$$
\left(g^{i j}(x, \varphi)\right)=\frac{1}{v(x)^{2}\left(1+v^{\prime}(x)^{2}\right)}\left(\begin{array}{cc}
v(x)^{2} & 0 \\
0 & 1+v^{\prime}(x)^{2}
\end{array}\right), \quad \mathrm{d} A=v \sqrt{1+\left(v^{\prime}\right)^{2}} \mathrm{~d} x \mathrm{~d} \varphi .
$$

Furthermore, we have the following formulae for the mean curvature $H$ and the Gauss curvature $K$ :

$$
H(x)=\frac{1}{2}\left[-\frac{v^{\prime \prime}(x)}{\left.\left(1+v^{\prime}(x)\right)^{2}\right)^{3 / 2}}+\frac{1}{v(x)\left(1+v^{\prime}(x)^{2}\right)^{1 / 2}}\right], \quad K(x)=-\frac{v^{\prime \prime}(x)}{v(x)\left(1+v^{\prime}(x)^{2}\right)^{2}} .
$$

In view of 2.1], 2.2] we obtain

$$
\begin{aligned}
W(v) & =\frac{\pi}{2} \int_{I}\left[-\frac{v^{\prime \prime}(x)}{\left(1+v^{\prime}(x)^{2}\right)^{3 / 2}}+\frac{1}{v(x)\left(1+v^{\prime}(x)^{2}\right)^{1 / 2}}\right]^{2} v(x) \sqrt{1+v^{\prime}(x)^{2}} \mathrm{~d} x \\
& =\frac{\pi}{2} \int_{I}\left[\frac{v(x) v^{\prime \prime}(x)^{2}}{\left(1+v^{\prime}(x)^{2}\right)^{5 / 2}}-2 \frac{v^{\prime \prime}(x)}{\left(1+v^{\prime}(x)^{2}\right)^{3 / 2}}+\frac{1}{v(x)\left(1+v^{\prime}(x)^{2}\right)^{1 / 2}}\right] \mathrm{d} x \\
& =\frac{\pi}{2} \int_{I}\left[\frac{v(x) v^{\prime \prime}(x)^{2}}{\left(1+v^{\prime}(x)^{2}\right)^{5 / 2}}+\frac{1}{v(x)\left(1+v^{\prime}(x)^{2}\right)^{1 / 2}}\right] \mathrm{d} x-\pi\left[\frac{v^{\prime}(x)}{\left(1+v^{\prime}(x)^{2}\right)^{1 / 2}}\right]_{x=-1}^{x=1},
\end{aligned}
$$

where we have written $W(v)$ instead of $W(\Gamma)$. Hence, for $v \in M$ we have

$$
W(v)=2 \pi \tilde{W}(v)-\pi\left[\frac{\beta_{r}}{\left(1+\beta_{r}^{2}\right)^{1 / 2}}-\frac{\beta_{l}}{\left(1+\beta_{l}^{2}\right)^{1 / 2}}\right]
$$

with the abbreviation

$$
\tilde{W}(v)=\frac{1}{4} \int_{I}\left[\frac{v(x) v^{\prime \prime}(x)^{2}}{\left(1+v^{\prime}(x)^{2}\right)^{5 / 2}}+\frac{1}{v(x)\left(1+v^{\prime}(x)^{2}\right)^{1 / 2}}\right] \mathrm{d} x .
$$


As a consequence, the first variation of $W$ in direction $\phi \in H_{0}^{2}(I)$ is given by

$$
\left\langle W^{\prime}(v), \phi\right\rangle=2 \pi\left\langle\tilde{W}^{\prime}(v), \phi\right\rangle,
$$

where

$$
\begin{aligned}
\left\langle\tilde{W}^{\prime}(v), \phi\right\rangle=\frac{\mathrm{d}}{\mathrm{d} \epsilon} \tilde{W}(v+ & \epsilon \phi)\left.\right|_{\epsilon=0}=\frac{1}{4} \int_{I}\left\{2 \frac{v v^{\prime \prime} \phi^{\prime \prime}}{\left(1+\left(v^{\prime}\right)^{2}\right)^{5 / 2}}+\frac{\left(v^{\prime \prime}\right)^{2} \phi}{\left(1+\left(v^{\prime}\right)^{2}\right)^{5 / 2}}\right. \\
& \left.-5 \frac{v v^{\prime}\left(v^{\prime \prime}\right)^{2} \phi^{\prime}}{\left(1+\left(v^{\prime}\right)^{2}\right)^{7 / 2}}-\frac{\phi}{v^{2}\left(1+\left(v^{\prime}\right)^{2}\right)^{1 / 2}}-\frac{v^{\prime} \phi^{\prime}}{v\left(1+\left(v^{\prime}\right)^{2}\right)^{3 / 2}}\right\} \mathrm{d} x .
\end{aligned}
$$

For later purposes it is convenient to write 2.4 in the form

$$
\left\langle\tilde{W}^{\prime}(v), \phi\right\rangle=\sum_{k=0}^{2} \int_{I} b_{k}\left(v, v^{\prime}, v^{\prime \prime}\right) \phi^{(k)} \mathrm{d} x
$$

where for $s_{0}>0, s_{1}, s_{2} \in \mathbb{R}$,

$$
\begin{aligned}
& b_{0}\left(s_{0}, s_{1}, s_{2}\right)=\frac{1}{4} \frac{s_{2}^{2}}{\left(1+s_{1}^{2}\right)^{5 / 2}}-\frac{1}{4} \frac{1}{s_{0}^{2}\left(1+s_{1}^{2}\right)^{1 / 2}}, \\
& b_{1}\left(s_{0}, s_{1}, s_{2}\right)=-\frac{5}{4} \frac{s_{0} s_{1} s_{2}^{2}}{\left(1+s_{1}^{2}\right)^{7 / 2}}-\frac{1}{4} \frac{s_{1}}{s_{0}\left(1+s_{1}^{2}\right)^{3 / 2}}, \\
& b_{2}\left(s_{0}, s_{1}, s_{2}\right)=\frac{1}{2} \frac{s_{0} s_{2}}{\left(1+s_{1}^{2}\right)^{5 / 2}} .
\end{aligned}
$$

Suppose that $v \in M$ satisfies

$$
v(x) \geqslant c_{1}, x \in \bar{I}, \quad\|v\|_{H^{2}} \leqslant C_{1}
$$

for some positive constants $c_{1}, C_{1}$. We define the functions $a_{k j}: I \rightarrow \mathbb{R}$ by

$$
a_{k j}:=\frac{\partial b_{k}}{\partial s_{j}}\left(v, v^{\prime}, v^{\prime \prime}\right), \quad 0 \leqslant k, j \leqslant 2 .
$$

In particular, $a_{22}=\frac{1}{2} v\left(1+\left(v^{\prime}\right)^{2}\right)^{-5 / 2}$, so that there exists $\delta=\delta\left(c_{1}, C_{1}\right)>0$ with

$$
a_{22}(x) \geqslant \delta, \quad x \in \bar{I} .
$$

Using the continuous embedding $H^{1}(I) \hookrightarrow C^{0}(\bar{I})$ and the elementary inequalities

$$
\|f\|_{L^{\infty}} \leqslant \epsilon\left\|f^{\prime}\right\|+C_{\epsilon}\|f\|, \quad\left\|f^{\prime}\right\| \leqslant \epsilon\|f\|_{H^{2}}+C_{\epsilon}\|f\|
$$

we deduce that there exists $\gamma=\gamma\left(c_{1}, C_{1}\right)>0$ such that

$$
B(v ; \phi, \phi) \geqslant \frac{\delta}{2}\|\phi\|_{H^{2}}^{2} \quad \forall \phi \in H^{2}(I)
$$

where

$$
B(v ; \phi, \psi):=\sum_{k, j=0}^{2} \int_{I} a_{k j} \phi^{(j)} \psi^{(k)} \mathrm{d} x+\gamma \int_{I} \phi \psi \mathrm{d} x .
$$


Let us now return to the Willmore flow problem and suppose that a family of surfaces $(\Gamma(t))_{t \in[0, T]}$ of the form 1.3$)$ evolves according to 1.2$)$. The normal velocity of $\Gamma(t)$ in the direction of $v$ is given by

$$
V=\frac{u_{t}}{\left(1+u_{x}^{2}\right)^{1 / 2}},
$$

where we will use $u_{x}$ rather than $u^{\prime}$ to denote a spatial derivative when functions of $x$ and $t$ are involved. Lemma A.1 in [8] implies

$$
\left\langle\tilde{W}^{\prime}(u(\cdot, t)), \phi\right\rangle=-\int_{I} u(\cdot, t) \phi\left(\Delta_{\Gamma} H(\cdot, t)+2 H(\cdot, t)^{3}-2 H(\cdot, t) K(\cdot, t)\right) \mathrm{d} x, \quad \phi \in C_{0}^{\infty}(I) .
$$

Therefore, multiplying 1.2 by $u(\cdot, t) \phi, \phi \in H_{0}^{2}(I)$, integrating over $I$ and taking into account (2.11) yields

$$
\int_{I} \frac{u(\cdot, t) u_{t}(\cdot, t) \phi}{\sqrt{1+u_{x}(\cdot, t)^{2}}} \mathrm{~d} x+\left\langle\tilde{W}^{\prime}(u(\cdot, t)), \phi\right\rangle=0 \quad \forall \phi \in H_{0}^{2}(I), t \in(0, T] .
$$

Note that if we make use of the formula 2.4 for $\left\langle\tilde{W}^{\prime}(u(\cdot, t)), \phi\right\rangle$ then only spatial derivatives up to second order of both $u(\cdot, t)$ and $\phi$ appear. As mentioned above we impose the boundary conditions

$$
u(-1, t)=\alpha_{l}, u(1, t)=\alpha_{r}, u_{x}(-1, t)=\beta_{l}, u_{x}(1, t)=\beta_{r}, \quad 0 \leqslant t \leqslant T .
$$

Finally, we assume

$$
u(x, 0)=u_{0}(x), \quad x \in I,
$$

for a given smooth positive function $u_{0}: \bar{I} \rightarrow \mathbb{R}$

REMARK 2.1 Combining (2.11) and (2.2) with the formulae

$$
\Delta_{\Gamma} H=\frac{1}{u \sqrt{1+u_{x}^{2}}} \partial_{x}\left(\frac{u}{\sqrt{1+u_{x}^{2}}} H_{x}\right), \quad H^{2}-K=\frac{1}{4}\left(\frac{u_{x x}}{\left(1+u_{x}^{2}\right)^{3 / 2}}+\frac{1}{u\left(1+u_{x}^{2}\right)^{1 / 2}}\right)^{2}
$$

we can show that the evolution law (1.2) is equivalent to the following quasilinear fourth order parabolic PDE for $u$ :

$$
\begin{aligned}
u_{t}= & \frac{1}{2 u}\left\{\frac{u}{\left(1+u_{x}^{2}\right)^{1 / 2}}\left(-\frac{u_{x x}}{\left(1+u_{x}^{2}\right)^{3 / 2}}+\frac{1}{u\left(1+u_{x}^{2}\right)^{1 / 2}}\right)_{x}\right\}_{x} \\
& +\frac{1}{4}\left(-\frac{u_{x x}}{1+u_{x}^{2}}+\frac{1}{u}\right)\left(\frac{u_{x x}}{\left(1+u_{x}^{2}\right)^{3 / 2}}+\frac{1}{u\left(1+u_{x}^{2}\right)^{1 / 2}}\right)^{2} \\
= & : L\left(u, u_{x}, u_{x x}, u_{x x x}, u_{x x x x}\right) \quad \text { in } I \times(0, T] .
\end{aligned}
$$

It is beyond the scope of this paper to prove an existence and uniqueness theorem for 2.15, 2.13), 2.14]. A local result could be obtained by linearizing $L$ around $u_{0}$ and by combining the linear theory for higher order parabolic equations with a fixed point argument. This would give the existence of a unique solution $u \in C^{4+\alpha}(\bar{I} \times[0, T])(\alpha \in(0,1))$ for some $T>0$ provided that $u_{0} \in C^{4+\alpha}(\bar{I})$ satisfies the compatibility conditions

$$
\begin{gathered}
u_{0}(-1)=\alpha_{l}, \quad u_{0}(1)=\alpha_{r}, \quad u_{0, x}(-1)=\beta_{l}, \quad u_{0, x}(1)=\beta_{r}, \\
L\left(u_{0}( \pm 1), u_{0, x}( \pm 1), u_{0, x x}( \pm 1), u_{0, x x x}( \pm 1), u_{0, x x x x}( \pm 1)\right)=0 .
\end{gathered}
$$


The definition of the parabolic Hölder spaces $C^{s}(\bar{I} \times[0, T])$ and a description of the compatibility conditions can be found in [11, II.3.1 and VI.3.2] respectively. By further strengthening the hypotheses on $u_{0}$ we may assume that there exist $0<c_{0}<C$ such that

$$
u(x, t) \geqslant c_{0},(x, t) \in \bar{I} \times[0, T], \quad \max _{0 \leqslant t \leqslant T}\left(\|u(\cdot, t)\|_{C^{4}}+\left\|u_{t}(\cdot, t)\right\|_{C^{4}}\right) \leqslant C .
$$

\section{Discretization and main result}

We now use 2.12) in order to discretize the problem in space. To this end let $-1=x_{0}<x_{1}<$ $\cdots<x_{N-1}<x_{N}=1, h_{j}=x_{j}-x_{j-1}$ and $h:=\max _{1 \leqslant j \leqslant N} h_{j}$. We make an inverse assumption of the form

$$
h \leqslant \eta h_{j}, \quad j=1, \ldots, N,
$$

where $\eta>0$ is independent of $h$. The space of $C^{1}$-finite elements is defined by

$$
X_{h}:=\left\{\phi_{h} \in C^{1}(\bar{I}) \mid \phi_{h \mid\left[x_{j-1}, x_{j}\right]} \in P_{3}, 1 \leqslant j \leqslant N\right\} \subset H^{2}(I) .
$$

Furthermore, we set $X_{h 0}:=X_{h} \cap H_{0}^{2}(I)$. There exists an interpolation operator $I_{h}: W^{4, p}(I) \rightarrow X_{h}$ with the property that $I_{h} v \in X_{h 0}$ for $v \in W^{4, p}(I) \cap H_{0}^{2}(I)$ and

$$
\sum_{i=0}^{2} h^{i}\left\|\left(v-I_{h} v\right)^{(i)}\right\|_{L^{p}} \leqslant C h^{4}\|v\|_{W^{4, p}} \quad \forall v \in W^{4, p}(I),
$$

where $1 \leqslant p \leqslant \infty$. Next, let us introduce a projection operator which is related to the first variation of $\tilde{W}$. Suppose that $u \in C^{4}(\bar{I})$ satisfies

$$
u(x) \geqslant c_{1}>0, x \in \bar{I}, \quad\|u\|_{C^{4}} \leqslant C_{1} .
$$

Then the coefficients $a_{k j}:=\frac{\partial b_{k}}{\partial s_{j}}\left(u, u^{\prime}, u^{\prime \prime}\right)$ (cf. 2.6) belong to $C^{2}(\bar{I})$ and there exists a constant $C=C\left(c_{1}, C_{1}\right)$ such that $\left\|a_{k j}\right\|_{C^{2}} \leqslant C$. For a given function $v \in H^{4}(I)$ we now introduce the projection $Q_{h} v \in X_{h}$ by requiring $\left(Q_{h} v\right)( \pm 1)=v( \pm 1),\left(Q_{h} v\right)^{\prime}( \pm 1)=v^{\prime}( \pm 1)$ and

$$
B\left(u ; v, \phi_{h}\right)=B\left(u ; Q_{h} v, \phi_{h}\right) \quad \forall \phi_{h} \in X_{h 0} .
$$

Note that the projection $Q_{h}$ depends on the function $u$. The existence of $Q_{h} v$ together with the error bound

$$
\left\|v-Q_{h} v\right\|_{H^{2}} \leqslant C h^{2}\|v\|_{H^{4}}
$$

follows from 3.2 and 2.9 . In order to obtain optimal error bounds we use a standard dual argument based on the solution of the boundary value problem

$$
\begin{aligned}
& \sum_{k, j=0}^{2}(-1)^{j} \frac{\mathrm{d}^{j}}{\mathrm{~d} x^{j}}\left(a_{k j} w^{(k)}\right)+\gamma w=v-Q_{h} v \quad \text { in } I, \\
& w(-1)=w(1)=w^{\prime}(-1)=w^{\prime}(1)=0 .
\end{aligned}
$$


The solvability of this problem along with the a priori estimate $\|w\|_{H^{4}} \leqslant C\left\|v-Q_{h} v\right\|$ is guaranteed by the regularity of the coefficients $a_{k j}$ and (2.9). Note that the constant $C$ again only depends on $c_{1}$ and $C_{1}$. Using $[3.2$, (3.4) and an interpolation argument we deduce that

$$
\left\|v-Q_{h} v\right\|+h\left\|\left(v-Q_{h} v\right)^{\prime}\right\|+h^{2}\left\|\left(v-Q_{h} v\right)^{\prime \prime}\right\| \leqslant C h^{4}\|v\|_{H^{4}} \quad \forall v \in H^{4}(I) .
$$

Our discrete problem reads: find a positive function $u_{h}: \bar{I} \times[0, T] \rightarrow \mathbb{R}$ such that $u_{h}(\cdot, t) \in X_{h}$ for $0 \leqslant t \leqslant T$ and

$$
\begin{gathered}
\int_{I} \frac{u_{h}(\cdot, t) u_{h t}(\cdot, t) \phi_{h}}{\sqrt{1+u_{h x}(\cdot, t)^{2}}} \mathrm{~d} x+\left\langle\tilde{W}^{\prime}\left(u_{h}(\cdot, t)\right), \phi_{h}\right\rangle=0 \quad \forall \phi_{h} \in X_{h 0}, 0<t \leqslant T, \\
u_{h}(-1, t)=\alpha_{l}, u_{h}(1, t)=\alpha_{r}, u_{h x}(-1, t)=\beta_{l}, u_{h x}(1, t)=\beta_{r}, \quad 0 \leqslant t \leqslant T, \\
u_{h}(\cdot, 0)=Q_{h 0} u_{0} .
\end{gathered}
$$

Here, $Q_{h 0}$ is the projection defined in 3.3 for $u=u_{0}$. Let us emphasize again that $\left\langle\tilde{W}^{\prime}\left(u_{h}(\cdot, t)\right), \phi_{h}\right\rangle$ is evaluated using the formula 2.4 . Problem $\sqrt{3.6}-(3.8)$ can be rewritten as a nonlinear system of ODEs which has a unique positive solution $u_{h}$ on some time interval $\left[0, T_{h}\right]$, $T_{h}>0$. Our main result are the following optimal error estimates for the semidiscrete scheme (3.6) -3.8 :

THEOREM 3.1 Suppose that 2.15, 2.13, 2.14 has a solution $u: \bar{I} \times[0, T] \rightarrow \mathbb{R}$ which satisfies the bounds 2.16. Then there exists $h_{0}>0$ such that for all $0<h \leqslant h_{0}$ the discrete solution $u_{h}$ exists on $[0, T]$ and

$$
\left(\int_{0}^{T}\left\|u_{t}-u_{h t}\right\|^{2} \mathrm{~d} t\right)^{1 / 2}+\sum_{i=0}^{2} h^{i} \max _{0 \leqslant t \leqslant T}\left\|\partial_{x}^{i}\left(u-u_{h}\right)(\cdot, t)\right\| \leqslant C h^{4} .
$$

The proof of this theorem will be carried out in Section 5. It relies on a decomposition of the error $e_{h}=u-u_{h}$ into

$$
e_{h}=\left(u-\hat{u}_{h}\right)+\left(\hat{u}_{h}-u_{h}\right)=: \rho_{h}+\theta_{h},
$$

where $\hat{u}_{h}$ denotes a suitable nonlinear projection of $u$ onto $X_{h}$. That projection is defined with the help of the elliptic part of the problem which is essentially given by the first variation of $\tilde{W}$ and satisfies the same error bounds as the interpolation operator (see Section 4). The remaining part $\theta_{h}$ is controlled using energy arguments and enjoys certain superconvergence properties. The procedure can be viewed as a nonlinear, fourth order version of a well-known argument by Wheeler for the heat equation (see [21]).

\section{A nonlinear projection}

The aim of this section is to introduce and analyze a nonlinear projection which is adapted to the first variation of $\tilde{W}$ and which will play a crucial role in proving our main result.

LEMma 4.1 Suppose that $u \in C^{4}(I)$ satisfies

$$
u(x) \geqslant c_{1}>0, x \in I, \quad\|u\|_{C^{4}} \leqslant C_{1},
$$


and let $Q_{h}$ be defined as above. Then there exist $0<h_{0} \leqslant 1$ and $K>0$ only depending on $c_{1}, C_{1}$ and $\eta$ such that for $0<h \leqslant h_{0}$ the problem

$$
\left\langle\tilde{W}^{\prime}\left(\hat{u}_{h}\right), \phi_{h}\right\rangle+\gamma \int_{I} \hat{u}_{h} \phi_{h} \mathrm{~d} x=\left\langle\tilde{W}^{\prime}(u), \phi_{h}\right\rangle+\gamma \int_{I} u \phi_{h} \mathrm{~d} x \quad \forall \phi_{h} \in X_{h 0}
$$

has a unique solution $\hat{u}_{h}$ in the set $\left\{v_{h} \in X_{h} \mid v_{h}-Q_{h} u \in X_{h 0},\left\|v_{h}-Q_{h} u\right\|_{H^{2}} \leqslant K h^{4}\right\}$. Furthermore, there exists $C=C\left(c_{1}, C_{1}, \eta\right)$ such that

$$
\left\|u-\hat{u}_{h}\right\|+h\left\|\left(u-\hat{u}_{h}\right)^{\prime}\right\|+h^{2}\left\|\left(u-\hat{u}_{h}\right)^{\prime \prime}\right\| \leqslant C h^{4}
$$

Proof. Let us define

$$
M_{h}:=\left\{v_{h} \in X_{h} \mid v_{h}-Q_{h} u \in X_{h 0},\left\|v_{h}-Q_{h} u\right\|_{H^{2}} \leqslant K h^{4}\right\}
$$

where the constant $K$ will be defined later. Using an inverse inequality, 3.2, 3.4 and the definition of $M_{h}$ we have, for $v_{h} \in M_{h}$,

$$
\begin{aligned}
\left\|v_{h}\right\|_{W^{2, \infty}} & \leqslant\left\|v_{h}-I_{h} u\right\|_{W^{2, \infty}}+\left\|u-I_{h} u\right\|_{W^{2, \infty}}+\|u\|_{W^{2, \infty}} \\
& \leqslant C h^{-1 / 2}\left\|v_{h}-I_{h} u\right\|_{H^{2}}+C h^{2}\|u\|_{W^{4, \infty}}+C_{1} \\
& \leqslant C h^{-1 / 2}\left(\left\|v_{h}-Q_{h} u\right\|_{H^{2}}+\left\|u-Q_{h} u\right\|_{H^{2}}+\left\|u-I_{h} u\right\|_{H^{2}}\right)+C \\
& \leqslant C+C K h^{7 / 2} \leqslant 2 C
\end{aligned}
$$

provided that $K h_{0}^{7 / 2} \leqslant 1$. Furthermore, since $\|f\|_{L^{\infty}} \leqslant\|f\|_{H^{1}}$ for $f \in H_{0}^{1}(I)$ we infer with the help of 3.5 and (4.1) that

$$
v_{h}(x) \geqslant u(x)-\left\|u-Q_{h} u\right\|_{H^{1}}-\left\|v_{h}-Q_{h} u\right\|_{H^{1}} \geqslant c_{1}-C h^{3}-K h_{0}^{4} \geqslant \frac{c_{1}}{2}, \quad x \in I,
$$

provided that $C h_{0}^{3} \leqslant c_{1} / 4$ and $K h_{0}^{4} \leqslant c_{1} / 4$. Using a Taylor expansion, 2.10 as well as 3.3 we may write, for $v_{h} \in M_{h}$,

$$
\begin{aligned}
& \left\langle\tilde{W}^{\prime}\left(v_{h}\right), \phi_{h}\right\rangle+\gamma \int_{I} v_{h} \phi_{h} \mathrm{~d} x-\left\langle\tilde{W}^{\prime}(u), \phi_{h}\right\rangle-\gamma \int_{I} u \phi_{h} \mathrm{~d} x \\
& \quad=\sum_{k=0}^{2} \int_{I}\left(b_{k}\left(v_{h}\right)-b_{k}(u)\right) \phi_{h}^{(k)} \mathrm{d} x+\gamma \int_{I}\left(v_{h}-u\right) \phi_{h} \mathrm{~d} x \\
& \quad=B\left(u ; v_{h}-u, \phi_{h}\right)+\left\langle R_{h}\left(u ; v_{h}\right), \phi_{h}\right\rangle=B\left(u ; v_{h}-Q_{h} u, \phi_{h}\right)+\left\langle R_{h}\left(u ; v_{h}\right), \phi_{h}\right\rangle,
\end{aligned}
$$

where we have abbreviated $b_{k}(u)=b_{k}\left(u, u^{\prime}, u^{\prime \prime}\right)$ etc. and where

$$
\begin{aligned}
& \left\langle R_{h}\left(u ; v_{h}\right), \phi_{h}\right\rangle \\
& \quad=\sum_{k, j, l=0}^{2} \int_{I} \int_{0}^{1}(1-\sigma) \frac{\partial^{2} b_{k}}{\partial s_{j} \partial s_{l}}\left(u+\sigma\left(v_{h}-u\right)\right)\left(v_{h}-u\right)^{(j)}\left(v_{h}-u\right)^{(l)} \phi_{h}^{(k)} \mathrm{d} \sigma \mathrm{d} x .
\end{aligned}
$$


It follows from $\sqrt{4.3}, \sqrt{4.4}$, the fact that $\partial^{2} b_{2} / \partial s_{2}^{2} \equiv 0$ and the continuous embedding $H^{1}(I) \hookrightarrow$ $C^{0}(\bar{I})$ that

$$
\begin{aligned}
\left|\left\langle R_{h}\left(u ; v_{h}\right), \phi_{h}\right\rangle\right| & \leqslant C\left\|v_{h}-u\right\|_{H^{2}}^{2}\left\|\phi_{h}\right\|_{H^{2}} \leqslant C\left(\left\|v_{h}-Q_{h} u\right\|_{H^{2}}^{2}+\left\|u-Q_{h} u\right\|_{H^{2}}^{2}\right)\left\|\phi_{h}\right\|_{H^{2}} \\
& \leqslant C\left(h^{4}+K^{2} h^{8}\right)\left\|\phi_{h}\right\|_{H^{2}}, \quad \phi_{h} \in X_{h 0},
\end{aligned}
$$

in view of 3.4 and the definition of $M_{h}$.

We define a mapping $F: M_{h} \rightarrow X_{h}$ as follows: for a given $v_{h} \in M_{h}$ we denote by $w_{h}=F\left(v_{h}\right)$ the solution of the following linear problem: $w_{h}-Q_{h} u \in X_{h 0}$ and

$$
B\left(u ; w_{h}, \phi_{h}\right)=B\left(u ; Q_{h} u, \phi_{h}\right)-\left\langle R_{h}\left(u ; v_{h}\right), \phi_{h}\right\rangle \quad \forall \phi_{h} \in X_{h 0} .
$$

We shall prove that $F$ has a fixed point provided that $h_{0}$ and $K$ are suitably chosen. To begin, let $v_{h} \in M_{h}, w_{h}=F\left(v_{h}\right)$. Inserting $\phi_{h}=w_{h}-Q_{h} u$ into (4.8) we obtain, with the help of (2.9) and 4.7,

and therefore

$$
\frac{\delta}{2}\left\|w_{h}-Q_{h} u\right\|_{H^{2}}^{2} \leqslant C\left(h^{4}+K^{2} h^{8}\right)\left\|w_{h}-Q_{h} u\right\|_{H^{2}},
$$

$$
\left\|w_{h}-Q_{h} u\right\|_{H^{2}} \leqslant \frac{2}{\delta} C h^{4}+\frac{2}{\delta} C K^{2} h^{8} \leqslant K h^{4},
$$

if we choose $K=4 C / \delta$ and $h_{0}>0$ so small that $K^{2} h_{0}^{4} \leqslant 1$. Hence we see that $w_{h} \in M_{h}$. Furthermore, by using similar arguments and choosing $h_{0}$ smaller if necessary one can show that $F$ is a contraction with respect to the norm in $H^{2}(I)$. Thus, Banach's fixed point theorem yields the existence of a unique solution $\hat{u}_{h} \in M_{h}$ of $F\left(\hat{u}_{h}\right)=\hat{u}_{h}$ which is the required solution of our problem in view of 4.5 . The bounds on $u-\hat{u}_{h}$ then follow from 3.2), 3.5. and the definition of $M_{h}$.

In the final part of this section we consider the above projection in the case that the function $u$ also depends on time. Suppose that $u: \bar{I} \times[0, T] \rightarrow \mathbb{R}$ is a smooth function satisfying 2.13 ) and $(2.16)$. For each $t \in[0, T]$ let $\hat{u}_{h}(\cdot, t) \in X_{h}$ be the nonlinear projection of $u(\cdot, t)$ defined in Lemma 4.1 . Hence,

$$
\sum_{i=0}^{2} h^{i} \max _{0 \leqslant t \leqslant T}\left\|\partial_{x}^{i}\left(u(\cdot, t)-\hat{u}_{h}(\cdot, t)\right)\right\| \leqslant C h^{4} .
$$

Our error analysis will also require estimates analogous to (4.9) for the time derivative $u_{t}-\hat{u}_{h t}$. Using an argument based on the implicit function theorem it can be shown that $\hat{u}_{h} \in C^{1}\left([0, T] ; H^{2}(I)\right)$. Abbreviating $\rho_{h}:=u-\hat{u}_{h}$ and using the calculations in 4.5 we may write 4.2 in the form

$$
\sum_{k, j=0}^{2} \int_{I} a_{k j}(\cdot, t) \partial_{x}^{j} \rho_{h}(\cdot, t) \phi_{h}^{(k)} \mathrm{d} x+\gamma \int_{I} \rho_{h}(\cdot, t) \phi_{h} \mathrm{~d} x=\left\langle R_{h}\left(u(\cdot, t) ; \hat{u}_{h}(\cdot, t)\right), \phi_{h}\right\rangle
$$

for all $\phi_{h} \in X_{h 0}$ and $0 \leqslant t \leqslant T$, where $a_{k j}(\cdot, t)=\frac{\partial b_{k}}{\partial s_{j}}\left(u(\cdot, t), u_{x}(\cdot, t), u_{x x}(\cdot, t)\right.$ ) (see $\left.\sqrt{2.6}\right)$ ). Differentiating with respect to time we obtain

$$
\sum_{k, j=0}^{2} \int_{I} a_{k j} \partial_{x}^{j} \rho_{h t} \phi_{h}^{(k)} \mathrm{d} x+\gamma \int_{I} \rho_{h t} \phi_{h} \mathrm{~d} x=-\sum_{k, j=0}^{2} \int_{I} a_{k j, t} \partial_{x}^{j} \rho_{h} \phi_{h}^{(k)} \mathrm{d} x+\partial_{t}\left(\left\langle R_{h}\left(u ; \hat{u}_{h}\right), \phi_{h}\right\rangle\right)
$$


for all $\phi_{h} \in X_{h 0}$ and $0 \leqslant t \leqslant T$. Recalling $\sqrt{4.6}$, the fact that $\partial^{2} b_{2} / \partial s_{2}^{2} \equiv 0$ as well as the continuous embedding $H^{1}(I) \hookrightarrow C^{0}(\bar{I})$ it is not difficult to verify that

$$
\left|\partial_{t}\left(\left\langle R_{h}\left(u ; \hat{u}_{h}\right), \phi_{h}\right\rangle\right)\right| \leqslant C\left\|\rho_{h}\right\|_{H^{2}}^{2}\left\|\phi_{h}\right\|_{H^{2}}+C\left\|\rho_{h t}\right\|_{H^{2}}\left\|\rho_{h}\right\|_{H^{2}}\left\|\phi_{h}\right\|_{H^{2}} .
$$

Inserting $\phi_{h}=I_{h} u_{t}(\cdot, t)-\hat{u}_{h t}(\cdot, t)$ into 4.10 and combining 4.9, 4.11 with estimates similar to those used above we derive

$$
\max _{0 \leqslant t \leqslant T}\left\|u_{t}(\cdot, t)-\hat{u}_{h t}(\cdot, t)\right\|_{H^{2}} \leqslant C h^{2}
$$

provided that $0<h \leqslant h_{0}$, where $h_{0}$ is sufficiently small. In order to obtain an optimal bound for $\left\|u_{t}(\cdot, t)-\hat{u}_{h t}(\cdot, t)\right\|$ we again employ a duality argument. For fixed $t \in[0, T]$ we consider the boundary value problem

$$
\begin{aligned}
& \sum_{k, j=0}^{2}(-1)^{j} \frac{\mathrm{d}^{j}}{\mathrm{~d} x^{j}}\left(a_{k j}(\cdot, t) w^{(k)}\right)+\gamma w=\rho_{h t}(\cdot, t) \quad \text { in } I, \\
& w(-1)=w(1)=w^{\prime}(-1)=w^{\prime}(1)=0 .
\end{aligned}
$$

This problem has a unique solution $w \in H^{4}(I) \cap H_{0}^{2}(I)$ satisfying

$$
\|w\|_{H^{4}} \leqslant C\left\|\rho_{h t}(\cdot, t)\right\|
$$

where $C$ is independent of $t \in[0, T]$. Multiplying the dual equation by $\rho_{h t}$, integrating by parts and recalling 4.10 yields

$$
\begin{aligned}
\int_{I} \rho_{h t}^{2} \mathrm{~d} x= & \sum_{k, j=0}^{2} \int_{I} a_{k j} \partial_{x}^{j} \rho_{h t}\left(w-I_{h} w\right)^{(k)} \mathrm{d} x+\gamma \int_{I} \rho_{h t}\left(w-I_{h} w\right) \mathrm{d} x \\
& -\sum_{k, j=0}^{2} \int_{I} a_{k j, t} \partial_{x}^{j} \rho_{h}\left(I_{h} w\right)^{(k)} \mathrm{d} x+\partial_{t}\left(\left\langle R_{h}\left(u ; \hat{u}_{h}\right), I_{h} w\right\rangle\right) \equiv I+I I+I I I+I V,
\end{aligned}
$$

where we have suppressed the $t$-variable. We infer from 4.12, 3.2 and 4.13 that

$$
|I|+|I I| \leqslant C\left\|\rho_{h t}\right\|_{H^{2}}\left\|w-I_{h} w\right\|_{H^{2}} \leqslant C h^{4}\|w\|_{H^{4}} \leqslant C h^{4}\left\|\rho_{h t}\right\| .
$$

Integration by parts implies

$$
\begin{aligned}
I I I & =-\sum_{k, j=0}^{2} \int_{I} a_{k j, t} \partial_{x}^{j} \rho_{h} w^{(k)} \mathrm{d} x+\sum_{k, j=0}^{2} \int_{I} a_{k j, t} \partial_{x}^{j} \rho_{h}\left(w-I_{h} w\right)^{(k)} \mathrm{d} x \\
& =-\sum_{k, j=0}^{2}(-1)^{j} \int_{I} \frac{\partial^{j}}{\partial x^{j}}\left(a_{k j, t} w^{(k)}\right) \rho_{h} \mathrm{~d} x+\sum_{k, j=0}^{2} \int_{I} a_{k j, t} \partial_{x}^{j} \rho_{h}\left(w-I_{h} w\right)^{(k)} \mathrm{d} x,
\end{aligned}
$$

and hence

$$
|I I I| \leqslant C\left\|\rho_{h}\right\|\|w\|_{H^{4}}+C h^{2}\left\|\rho_{h}\right\|_{H^{2}}\|w\|_{H^{4}} \leqslant C h^{4}\left\|\rho_{h t}\right\| .
$$


Finally,

$$
|I V| \leqslant C h^{4}\left\|I_{h} w\right\|_{H^{2}} \leqslant C h^{4}\left(\|w\|_{H^{2}}+\left\|w-I_{h} w\right\|_{H^{2}}\right) \leqslant C h^{4}\left\|\rho_{h t}\right\| .
$$

As a result we obtain $\max _{0 \leqslant t \leqslant T}\left\|\rho_{h t}\right\| \leqslant C h^{4}$ and combining this estimate with 4.12 finally implies

$$
\sum_{i=0}^{2} h^{i} \max _{0 \leqslant t \leqslant T}\left\|\partial_{x}^{i}\left(u_{t}(\cdot, t)-\hat{u}_{h t}(\cdot, t)\right)\right\| \leqslant C h^{4}
$$

\section{Proof of Theorem 3.1}

As already mentioned above, the proof of Theorem 3.1 relies on the decomposition 3.9 ) of the error $e_{h}=u-u_{h}$. Since the projection error $\rho_{h}=u-\hat{u}_{h}$ has been treated in the previous section, it remains to analyze the error $\theta_{h}=\hat{u}_{h}-u_{h}$. Note that 3.8 and Lemma 4.1 imply that

$$
\left\|\theta_{h}(\cdot, 0)\right\|_{H^{2}}=\left\|\hat{u}_{h}(\cdot, 0)-Q_{h 0} u_{0}\right\|_{H^{2}} \leqslant C h^{4} .
$$

Recalling 2.16) there exists $C_{0}>0$ such that

$$
c_{0} \leqslant u(x, t) \leqslant C_{0}, \quad\left|u_{x}(x, t)\right|,\left|u_{x x}(x, t)\right| \leqslant C_{0} \quad \forall(x, t) \in \bar{I} \times[0, T] .
$$

By choosing $T_{h}>0$ smaller if necessary we may assume that

$$
c_{0} / 2 \leqslant u_{h}(x, t) \leqslant 2 C_{0}, \quad\left|u_{h x}(x, t)\right|,\left|u_{h x x}(x, t)\right| \leqslant 2 C_{0} \quad \forall(x, t) \in \bar{I} \times\left[0, T_{h}\right] .
$$

Next, let us define

$$
\begin{aligned}
& \hat{T}_{h}:=\sup \left\{t \in[0, T] \mid u_{h} \text { solves } 3.6-3.8\right) \text { on }[0, t] \text { and } c_{0} / 2 \leqslant u_{h}(x, s) \leqslant 2 C_{0} \text {, } \\
& \left.\left|u_{h x}(x, s)\right|,\left|u_{h x x}(x, s)\right| \leqslant 2 C_{0} \forall(x, s) \in \bar{I} \times[0, t]\right\} .
\end{aligned}
$$

Clearly, $\hat{T}_{h} \geqslant T_{h}>0$. Our aim is to show that $\hat{T}_{h}=T$ for small $h$. By definition of $\hat{T}_{h}$ we have

$$
c_{0} / 2 \leqslant u_{h}(x, t) \leqslant 2 C_{0}, \quad\left|u_{h x}(x, t)\right|,\left|u_{h x x}(x, t)\right| \leqslant 2 C_{0} \quad \forall(x, t) \in \bar{I} \times\left[0, \hat{T}_{h}\right) .
$$

In what follows, we shall denote by $C$ a constant which may depend on $c_{0}, C_{0}, T, \eta$ (see (3.1)) and on the constant appearing in 2.16. In order to shorten the formulae we shall omit $\mathrm{d} x$ in the integrals.

Taking the difference of 2.12) and (3.6) and recalling the definition of $\hat{u}_{h}$ we derive

$$
\begin{aligned}
& \int_{I} \frac{u_{h}\left(\hat{u}_{h t}-u_{h t}\right) \phi_{h}}{\sqrt{1+u_{h x}^{2}}}+\left\langle\tilde{W}^{\prime}\left(\hat{u}_{h}\right), \phi_{h}\right\rangle-\left\langle\tilde{W}^{\prime}\left(u_{h}\right), \phi_{h}\right\rangle \\
& \quad=\int_{I}\left(\frac{u_{h}}{\sqrt{1+u_{h x}^{2}}}-\frac{u}{\sqrt{1+u_{x}^{2}}}\right) u_{t} \phi_{h}+\int_{I} \frac{u_{h}\left(\hat{u}_{h t}-u_{t}\right) \phi_{h}}{\sqrt{1+u_{h x}^{2}}}+\gamma \int_{I}\left(u-\hat{u}_{h}\right) \phi_{h}
\end{aligned}
$$


for all $\phi_{h} \in X_{h 0}$ and $0 \leqslant t<\hat{T}_{h}$. Using $\phi_{h}=\left(\hat{u}_{h t}-u_{h t}\right)(\cdot, t)=\theta_{h t}(\cdot, t) \in X_{h 0}$ in 5.5 we obtain

$$
\begin{aligned}
& c_{2}\left\|\theta_{h t}\right\|^{2}+\left\langle\tilde{W}^{\prime}\left(\hat{u}_{h}\right), \theta_{h t}\right\rangle-\left\langle\tilde{W}^{\prime}\left(u_{h}\right), \theta_{h t}\right\rangle \\
& \leqslant \int_{I}\left(\frac{1}{\sqrt{1+u_{h x}^{2}}}-\frac{1}{\sqrt{1+u_{x}^{2}}}\right) u u_{t} \theta_{h t}-\int_{I} \frac{e_{h} u_{t} \theta_{h t}}{\sqrt{1+u_{h x}^{2}}}-\int_{I} \frac{u_{h} \rho_{h t} \theta_{h t}}{\sqrt{1+u_{h x}^{2}}}+\gamma \int_{I} \rho_{h} \theta_{h t} \\
& \equiv I+I I+I I I+I V .
\end{aligned}
$$

Here, $c_{2}=c_{2}\left(c_{0}, C_{0}\right)>0$ in view of (5.4). Clearly, 22.16, (5.4, 4.9) and 4.14) imply

$$
|I I|+|I I I|+|I V| \leqslant C\left(\left\|e_{h}\right\|+\left\|\rho_{h t}\right\|+\left\|\rho_{h}\right\|\right)\left\|\theta_{h t}\right\| \leqslant \epsilon\left\|\theta_{h t}\right\|^{2}+C_{\epsilon}\left(h^{8}+\left\|\theta_{h}\right\|^{2}\right) .
$$

Next, let us write

$$
\frac{1}{\sqrt{1+u_{h x}^{2}}}-\frac{1}{\sqrt{1+u_{x}^{2}}}=-\frac{u_{x}}{\left(1+u_{x}^{2}\right)^{3 / 2}}\left(u_{h x}-u_{x}\right)+R=\frac{u_{x} e_{h x}}{\left(1+u_{x}^{2}\right)^{3 / 2}}+R,
$$

where $|R| \leqslant C\left|e_{h x}\right|^{2}$. Abbreviating $f=u u_{t} u_{x} /\left(1+u_{x}^{2}\right)^{3 / 2}$ we have

$$
I=\int_{I} f e_{h x} \theta_{h t}+\int_{I} u u_{t} R \theta_{h t} \equiv I_{1}+I_{2}
$$

Integration by parts yields

$$
I_{1}=-\int_{I} f_{x} e_{h} \theta_{h t}-\int_{I} f e_{h} \theta_{h t x}=-\int_{I} f_{x} e_{h} \theta_{h t}-\frac{\mathrm{d}}{\mathrm{d} t} \int_{I} f e_{h} \theta_{h x}+\int_{I} f_{t} e_{h} \theta_{h x}+\int_{I} f e_{h t} \theta_{h x} .
$$

As a result,

$$
\begin{aligned}
I_{1} & \leqslant-\frac{\mathrm{d}}{\mathrm{d} t} \int_{I} f e_{h} \theta_{h x}+C\left(\left\|e_{h}\right\|\left\|\theta_{h t}\right\|+\left\|e_{h}\right\|\left\|\theta_{h x}\right\|+\left\|e_{h t}\right\|\left\|\theta_{h x}\right\|\right) \\
& \leqslant-\frac{\mathrm{d}}{\mathrm{d} t} \int_{I} f e_{h} \theta_{h x}+\epsilon\left\|\theta_{h t}\right\|^{2}+C_{\epsilon}\left(h^{8}+\left\|\theta_{h}\right\|_{H^{1}}^{2}\right)
\end{aligned}
$$

in view of 4.9 and 4.14. Note in particular that $\left\|f_{t}\right\|_{C^{0}} \leqslant C$ follows from 2.16 and by differentiating 2.15) with respect to $t$. Furthermore, using also (5.4) we obtain

$$
I_{2} \leqslant C \int_{I}\left(\left|\rho_{h x}\right|^{2}+\left|\theta_{h x}\right|^{2}\right)\left|\theta_{h t}\right| \leqslant C\left(\left\|\rho_{h}\right\|_{H^{2}}^{2}+\left\|\theta_{h}\right\|_{H^{1}}\right)\left\|\theta_{h t}\right\| \leqslant \epsilon\left\|\theta_{h t}\right\|^{2}+C_{\epsilon}\left(h^{8}+\left\|\theta_{h}\right\|_{H^{1}}^{2}\right) .
$$

In conclusion we have

$$
I+I I+I I I+I V \leqslant-\frac{\mathrm{d}}{\mathrm{d} t} \int_{I} f e_{h} \theta_{h x}+\epsilon\left\|\theta_{h t}\right\|^{2}+C_{\epsilon}\left(h^{8}+\left\|\theta_{h}\right\|_{H^{1}}^{2}\right) .
$$

Let us next turn to the left hand side of 5.6. Clearly,

$$
\begin{aligned}
\left\langle\tilde{W}^{\prime}\left(\hat{u}_{h}\right), \theta_{h t}\right\rangle-\left\langle\tilde{W}^{\prime}\left(u_{h}\right), \theta_{h t}\right\rangle & \\
& =\left\langle\tilde{W}^{\prime}\left(\hat{u}_{h}\right), \hat{u}_{h t}\right\rangle-\left\langle\tilde{W}^{\prime}\left(\hat{u}_{h}\right), u_{h t}\right\rangle-\left\langle\tilde{W}^{\prime}\left(u_{h}\right), \hat{u}_{h t}\right\rangle+\left\langle\tilde{W}^{\prime}\left(u_{h}\right), u_{h t}\right\rangle .
\end{aligned}
$$


To begin, recalling 2.3, 2.4), we have

$$
\begin{aligned}
\left\langle\tilde{W}^{\prime}\left(\hat{u}_{h}\right), \hat{u}_{h t}\right\rangle & =\frac{\mathrm{d}}{\mathrm{d} t} \tilde{W}\left(\hat{u}_{h}\right) \\
& =\frac{1}{4} \frac{\mathrm{d}}{\mathrm{d} t} \int_{I} \frac{\hat{u}_{h} \hat{u}_{h x x}^{2}}{\left(1+\hat{u}_{h x}^{2}\right)^{5 / 2}}-\frac{1}{4} \int_{I} \frac{\hat{u}_{h t}}{\hat{u}_{h}^{2}\left(1+\hat{u}_{h x}^{2}\right)^{1 / 2}}-\frac{1}{4} \int_{I} \frac{\hat{u}_{h x} \hat{u}_{h t x}}{\hat{u}_{h}\left(1+\hat{u}_{h x}^{2}\right)^{3 / 2}} \\
& =\frac{1}{2} \frac{\mathrm{d}}{\mathrm{d} t} \int_{I} \frac{\hat{u}_{h} \hat{u}_{h x x}^{2}}{\left(1+\hat{u}_{h x}^{2}\right)^{5 / 2}}+\int_{I}\left(\hat{u}_{h t} A_{1}+\hat{u}_{h t x} B_{1}+\hat{u}_{h t x x} C_{1}\right),
\end{aligned}
$$

where

$$
\begin{aligned}
A_{1} & =-\frac{1}{4} \frac{\hat{u}_{h x x}^{2}}{\left(1+\hat{u}_{h x}^{2}\right)^{5 / 2}}-\frac{1}{4} \frac{1}{\hat{u}_{h}^{2}\left(1+\hat{u}_{h x}^{2}\right)^{1 / 2}}, \\
B_{1} & =\frac{5}{4} \frac{\hat{u}_{h} \hat{u}_{h x} \hat{u}_{h x x}^{2}}{\left(1+\hat{u}_{h x}^{2}\right)^{7 / 2}}-\frac{1}{4} \frac{\hat{u}_{h x}}{\hat{u}_{h}\left(1+\hat{u}_{h x}^{2}\right)^{3 / 2}}, \\
C_{1} & =-\frac{1}{2} \frac{\hat{u}_{h} \hat{u}_{h x x}}{\left(1+\hat{u}_{h x}^{2}\right)^{5 / 2}} .
\end{aligned}
$$

Next, again by 2.4,

$$
\begin{aligned}
\left\langle\tilde{W}^{\prime}\left(\hat{u}_{h}\right), u_{h t}\right\rangle= & \frac{1}{2} \int_{I} \frac{\hat{u}_{h} \hat{u}_{h x x} u_{h t x x}}{\left(1+\hat{u}_{h x}^{2}\right)^{5 / 2}}+\frac{1}{4} \int_{I} \frac{\hat{u}_{h x x}^{2} u_{h t}}{\left(1+\hat{u}_{h x}^{2}\right)^{5 / 2}} \\
& -\frac{5}{4} \int_{I} \frac{\hat{u}_{h} \hat{u}_{h x} \hat{u}_{h x x}^{2} u_{h t x}}{\left(1+\hat{u}_{h x}^{2}\right)^{7 / 2}}-\frac{1}{4} \int_{I} \frac{u_{h t}}{\hat{u}_{h}^{2}\left(1+\hat{u}_{h x}^{2}\right)^{1 / 2}}-\frac{1}{4} \int_{I} \frac{\hat{u}_{h x} u_{h t x}}{\hat{u}_{h}\left(1+\hat{u}_{h x}^{2}\right)^{3 / 2}} \\
= & \frac{1}{4} \frac{\mathrm{d}}{\mathrm{d} t} \int_{I}\left\{2 \frac{\hat{u}_{h} \hat{u}_{h x x} u_{h x x}}{\left(1+\hat{u}_{h x}^{2}\right)^{5 / 2}}+\frac{u_{h} \hat{u}_{h x x}^{2}}{\left(1+\hat{u}_{h x}^{2}\right)^{5 / 2}}-5 \frac{\hat{u}_{h} \hat{u}_{h x} \hat{u}_{h x x}^{2}\left(u_{h x}-\hat{u}_{h x}\right)}{\left(1+\hat{u}_{h x}^{2}\right)^{7 / 2}}-\frac{\hat{u}_{h x}\left(u_{h x}-\hat{u}_{h x}\right)}{\hat{u}_{h}\left(1+\hat{u}_{h x}^{2}\right)^{3 / 2}}\right. \\
& \left.+\frac{1}{u_{h}\left(1+\hat{u}_{h x}^{2}\right)^{1 / 2}}\right\}+\frac{1}{4} \int_{I} \frac{u_{h t}-\hat{u}_{h t}}{\left(1+\hat{u}_{h x}^{2}\right)^{1 / 2}}\left(\frac{1}{u_{h}^{2}}-\frac{1}{\hat{u}_{h}^{2}}\right)+\int_{I}\left(\hat{u}_{h t} A_{2}+\hat{u}_{h t x} B_{2}+\hat{u}_{h t x x} C_{2}\right),
\end{aligned}
$$

where

$$
\begin{aligned}
A_{2}= & -\frac{1}{2} \frac{\hat{u}_{h x x} u_{h x x}}{\left(1+\hat{u}_{h x}^{2}\right)^{5 / 2}}+\frac{5}{4} \frac{\hat{u}_{h x} \hat{u}_{h x x}^{2}\left(u_{h x}-\hat{u}_{h x}\right)}{\left(1+\hat{u}_{h x}^{2}\right)^{7 / 2}}-\frac{1}{4} \frac{\hat{u}_{h x}\left(u_{h x}-\hat{u}_{h x}\right)}{\hat{u}_{h}^{2}\left(1+\hat{u}_{h x}^{2}\right)^{3 / 2}} \\
& +\frac{1}{4} \frac{1}{\left(1+\hat{u}_{h x}^{2}\right)^{1 / 2}}\left(\frac{1}{u_{h}^{2}}-\frac{1}{\hat{u}_{h}^{2}}\right), \\
B_{2}= & \frac{5}{2} \frac{\hat{u}_{h} \hat{u}_{h x} \hat{u}_{h x x} u_{h x x}}{\left(1+\hat{u}_{h x}^{2}\right)^{7 / 2}}+\frac{5}{4} \frac{u_{h} \hat{u}_{h x} \hat{u}_{h x x}^{2}}{\left(1+\hat{u}_{h x}^{2}\right)^{7 / 2}}+\frac{5}{4} \frac{\hat{u}_{h} \hat{u}_{h x x}^{2}\left(u_{h x}-\hat{u}_{h x}\right)}{\left(1+\hat{u}_{h x}^{2}\right)^{7 / 2}} \\
& -\frac{35}{4} \frac{\hat{u}_{h} \hat{u}_{h x}^{2} \hat{u}_{h x x}^{2}\left(u_{h x}-\hat{u}_{h x}\right)}{\left(1+\hat{u}_{h x}^{2}\right)^{9 / 2}}-\frac{5}{4} \frac{\hat{u}_{h} \hat{u}_{h x} \hat{u}_{h x x}^{2}}{\left(1+\hat{u}_{h x}^{2}\right)^{7 / 2}}+\frac{1}{4} \frac{\hat{u}_{h x}}{u_{h}\left(1+\hat{u}_{h x}^{2}\right)^{3 / 2}} \\
& -\frac{1}{4} \frac{\hat{u}_{h x}}{\hat{u}_{h}\left(1+\hat{u}_{h x}^{2}\right)^{3 / 2}}+\frac{1}{4} \frac{u_{h x}-\hat{u}_{h x}}{\hat{u}_{h}\left(1+\hat{u}_{h x}^{2}\right)^{3 / 2}}-\frac{3}{4} \frac{\hat{u}_{h x}^{2}\left(u_{h x}-\hat{u}_{h x}\right)}{\hat{u}_{h}\left(1+\hat{u}_{h x}^{2}\right)^{5 / 2}},
\end{aligned}
$$


and

$$
C_{2}=-\frac{1}{2} \frac{\hat{u}_{h} u_{h x x}}{\left(1+\hat{u}_{h x}^{2}\right)^{5 / 2}}-\frac{1}{2} \frac{u_{h} \hat{u}_{h x x}}{\left(1+\hat{u}_{h x}^{2}\right)^{5 / 2}}+\frac{5}{2} \frac{\hat{u}_{h} \hat{u}_{h x} \hat{u}_{h x x}\left(u_{h x}-\hat{u}_{h x}\right)}{\left(1+\hat{u}_{h x}^{2}\right)^{7 / 2}} .
$$

Finally,

$$
\left\langle\tilde{W}^{\prime}\left(u_{h}\right), \hat{u}_{h t}\right\rangle=\int_{I}\left(\hat{u}_{h t} A_{3}+\hat{u}_{h t x} B_{3}+\hat{u}_{h t x x} C_{3}\right),
$$

where

$$
\begin{aligned}
A_{3} & =\frac{1}{4} \frac{u_{h x x}^{2}}{\left(1+u_{h x}^{2}\right)^{5 / 2}}-\frac{1}{4} \frac{1}{u_{h}^{2}\left(1+u_{h x}^{2}\right)^{1 / 2}}, \\
B_{3} & =-\frac{5}{4} \frac{u_{h} u_{h x} u_{h x x}^{2}}{\left(1+u_{h x}^{2}\right)^{7 / 2}}-\frac{1}{4} \frac{u_{h x}}{u_{h}\left(1+u_{h x}^{2}\right)^{3 / 2}}, \\
C_{3} & =\frac{1}{2} \frac{u_{h} u_{h x x}}{\left(1+u_{h x}^{2}\right)^{5 / 2}}
\end{aligned}
$$

while

$$
\left\langle\tilde{W}^{\prime}\left(u_{h}\right), u_{h t}\right\rangle=\frac{1}{4} \frac{\mathrm{d}}{\mathrm{d} t} \int_{I}\left\{\frac{u_{h} u_{h x x}^{2}}{\left(1+u_{h x}^{2}\right)^{5 / 2}}+\frac{1}{u_{h}\left(1+u_{h x}^{2}\right)^{1 / 2}}\right\} .
$$

Combining the above expressions we derive

$$
\begin{aligned}
\left\langle\tilde{W}^{\prime}\left(\hat{u}_{h}\right),\right. & \left.\theta_{h t}\right\rangle-\left\langle\tilde{W}^{\prime}\left(u_{h}\right), \theta_{h t}\right\rangle \\
= & \psi^{\prime}(t)+\frac{1}{4} \int_{I} \frac{\hat{u}_{h t}-u_{h t}}{\left(1+\hat{u}_{h x}^{2}\right)^{1 / 2}}\left(\frac{1}{u_{h}^{2}}-\frac{1}{\hat{u}_{h}^{2}}\right)+\int_{I}\left(\hat{u}_{h t} A+\hat{u}_{h t x} B+\hat{u}_{h t x x} C\right),
\end{aligned}
$$

where

$$
\begin{aligned}
\psi(t)= & \frac{1}{4} \int_{I}\left\{2 \frac{\hat{u}_{h} \hat{u}_{h x x}^{2}}{\left(1+\hat{u}_{h x}^{2}\right)^{5 / 2}}-2 \frac{\hat{u}_{h} \hat{u}_{h x x} u_{h x x}}{\left(1+\hat{u}_{h x}^{2}\right)^{5 / 2}}-\frac{u_{h} \hat{u}_{h x x}^{2}}{\left(1+\hat{u}_{h x}^{2}\right)^{5 / 2}}+5 \frac{\hat{u}_{h} \hat{u}_{h x} \hat{u}_{h x x}^{2}\left(u_{h x}-\hat{u}_{h x}\right)}{\left(1+\hat{u}_{h x}^{2}\right)^{7 / 2}}\right. \\
& \left.+\frac{\hat{u}_{h x}\left(u_{h x}-\hat{u}_{h x}\right)}{\hat{u}_{h}\left(1+\hat{u}_{h x}^{2}\right)^{3 / 2}}-\frac{1}{u_{h}\left(1+\hat{u}_{h x}^{2}\right)^{1 / 2}}+\frac{u_{h} u_{h x x}^{2}}{\left(1+u_{h x}^{2}\right)^{5 / 2}}+\frac{1}{u_{h}\left(1+u_{h x}^{2}\right)^{1 / 2}}\right\}
\end{aligned}
$$

and

$$
A=A_{1}-A_{2}-A_{3}, \quad B=B_{1}-B_{2}-B_{3}, \quad C=C_{1}-C_{2}-C_{3} .
$$

To begin, let us rewrite $\psi$ as follows:

$$
\begin{aligned}
\psi(t)= & \frac{1}{4} \int_{I}\left\{\left(\frac{u_{h x x}}{\left(1+u_{h x}^{2}\right)^{3 / 2}}-\frac{\hat{u}_{h}}{u_{h}} \frac{1+u_{h x}^{2}}{1+\hat{u}_{h x}^{2}} \frac{\hat{u}_{h x x}}{\left(1+\hat{u}_{h x}^{2}\right)^{3 / 2}}\right)^{2} u_{h}\left(1+u_{h x}^{2}\right)^{1 / 2}\right. \\
& -\frac{\hat{u}_{h} \hat{u}_{h x x}^{2}}{\left(1+\hat{u}_{h x}^{2}\right)^{5}}\left(\left(1+u_{h x}^{2}\right)^{5 / 2}-\left(1+\hat{u}_{h x}^{2}\right)^{5 / 2}-5 \hat{u}_{h x}\left(1+\hat{u}_{h x}^{2}\right)^{3 / 2}\left(u_{h x}-\hat{u}_{h x}\right)\right) \\
& +\frac{\hat{u}_{h x x}^{2}}{\left(1+\hat{u}_{h x}^{2}\right)^{5 / 2}}\left(\frac{\left(1+u_{h x}^{2}\right)^{5 / 2}}{\left(1+\hat{u}_{h x}^{2}\right)^{5 / 2}} \frac{\hat{u}_{h}}{u_{h}}-1\right)\left(u_{h}-\hat{u}_{h}\right)+\frac{\hat{u}_{h x}\left(u_{h x}-\hat{u}_{h x}\right)}{\left(1+\hat{u}_{h x}^{2}\right)^{3 / 2}}\left(\frac{1}{\hat{u}_{h}}-\frac{1}{u_{h}}\right) \\
& \left.+\frac{1}{u_{h}}\left(\frac{1}{\left(1+u_{h x}^{2}\right)^{1 / 2}}-\frac{1}{\left(1+\hat{u}_{h x}^{2}\right)^{1 / 2}}+\frac{\hat{u}_{h x}\left(u_{h x}-\hat{u}_{h x}\right)}{\left(1+\hat{u}_{h x}^{2}\right)^{3 / 2}}\right)\right\} .
\end{aligned}
$$


We claim that there exists $\mu>0$ such that

$$
\psi(t) \geqslant \mu\left\|\theta_{h}(\cdot, t)\right\|_{H^{2}}^{2}-C\left\|\theta_{h}(\cdot, t)\right\|_{H^{1}}^{2}, \quad 0 \leqslant t<T_{h} .
$$

To see this, let us examine the first term in the definition of $\psi$ in detail. Clearly,

$$
\begin{aligned}
& \frac{u_{h x x}}{\left(1+u_{h x}^{2}\right)^{3 / 2}}-\frac{\hat{u}_{h}}{u_{h}} \frac{1+u_{h x}^{2}}{1+\hat{u}_{h x}^{2}} \frac{\hat{u}_{h x x}}{\left(1+\hat{u}_{h x}^{2}\right)^{3 / 2}} \\
& =\frac{-\theta_{h x x}}{\left(1+u_{h x}^{2}\right)^{3 / 2}}+\hat{u}_{h x x}\left(\frac{1}{\left(1+u_{h x}^{2}\right)^{3 / 2}}-\frac{1}{\left(1+\hat{u}_{h x}^{2}\right)^{3 / 2}}+\frac{u_{h}\left(1+\hat{u}_{h x}^{2}\right)-\hat{u}_{h}\left(1+u_{h x}^{2}\right)}{u_{h}\left(1+\hat{u}_{h x}^{2}\right)^{5 / 2}}\right) .
\end{aligned}
$$

The elementary inequality $(a+b)^{2} \geqslant \frac{1}{2} a^{2}-b^{2}$ together with 5.4 implies that

$$
\begin{aligned}
\frac{1}{4} \int_{I}\left(\frac{u_{h x x}}{\left(1+u_{h x}^{2}\right)^{3 / 2}}\right. & \left.-\frac{\hat{u}_{h}}{u_{h}} \frac{1+u_{h x}^{2}}{1+\hat{u}_{h x}^{2}} \frac{\hat{u}_{h x x}}{\left(1+\hat{u}_{h x}^{2}\right)^{3 / 2}}\right)^{2} u_{h}\left(1+u_{h x}^{2}\right)^{1 / 2} \\
& \geqslant \frac{1}{16} \frac{c_{0}}{\left(1+4 C_{0}^{2}\right)^{3}}\left\|\theta_{h x x}\right\|^{2}-C\left\|\hat{u}_{h x x}\right\|_{L^{\infty}}^{2}\left\|\theta_{h}\right\|_{H^{1}}^{2} \geqslant \mu\left\|\theta_{h}\right\|_{H^{2}}^{2}-C\|\theta\|_{H^{1}}^{2},
\end{aligned}
$$

where we used the inequality $\|\phi\|_{H^{2}} \leqslant C\left\|\phi^{\prime \prime}\right\|$ for $\phi \in H_{0}^{2}(I)$ and the fact that $\left\|\hat{u}_{h}\right\|_{W^{2, \infty}} \leqslant C$. Estimating the remaining terms by $C\left\|\theta_{h}\right\|_{H^{1}}^{2}$ yields 5.14 .

Next, a long but straightforward calculation gives

$$
\begin{aligned}
& A=-\frac{1}{4} \frac{\left(\hat{u}_{h x x}-u_{h x x}\right)^{2}}{\left(1+\hat{u}_{h x}^{2}\right)^{5 / 2}}-\frac{u_{h x x}^{2}}{4}\left\{\frac{1}{\left(1+u_{h x}^{2}\right)^{5 / 2}}-\frac{1}{\left(1+\hat{u}_{h x}^{2}\right)^{5 / 2}}+5 \frac{\hat{u}_{h x}\left(u_{h x}-\hat{u}_{h x}\right)}{\left(1+\hat{u}_{h x}^{2}\right)^{7 / 2}}\right\} \\
& +\frac{5}{4} \frac{\hat{u}_{h x}\left(u_{h x x}^{2}-\hat{u}_{h x x}^{2}\right)\left(u_{h x}-\hat{u}_{h x}\right)}{\left(1+\hat{u}_{h x}^{2}\right)^{7 / 2}}+\frac{1}{4} \frac{\hat{u}_{h x}\left(u_{h x}-\hat{u}_{h x}\right)}{\left(1+\hat{u}_{h x}^{2}\right)^{3 / 2}}\left(\frac{1}{\hat{u}_{h}^{2}}-\frac{1}{u_{h}^{2}}\right) \\
& +\frac{1}{4 u_{h}^{2}}\left\{\frac{1}{\left(1+u_{h x}^{2}\right)^{1 / 2}}-\frac{1}{\left(1+\hat{u}_{h x}^{2}\right)^{1 / 2}}+\frac{\hat{u}_{h x}\left(u_{h x}-\hat{u}_{h x}\right)}{\left(1+\hat{u}_{h x}^{2}\right)^{3 / 2}}\right\}, \\
& B=\frac{5}{2} \frac{\hat{u}_{h x x}\left(\hat{u}_{h x x}-u_{h x x}\right)\left(\hat{u}_{h} \hat{u}_{h x}-u_{h} u_{h x}\right)}{\left(1+\hat{u}_{h x}^{2}\right)^{7 / 2}} \\
& +\frac{5}{4} \frac{u_{h} u_{h x}\left(\hat{u}_{h x x}-u_{h x x}\right)^{2}}{\left(1+\hat{u}_{h x}^{2}\right)^{7 / 2}}+\frac{5}{4} \frac{\hat{u}_{h x x}^{2}\left(\hat{u}_{h}-u_{h}\right)\left(\hat{u}_{h x}-u_{h x}\right)}{\left(1+\hat{u}_{h x}^{2}\right)^{7 / 2}} \\
& +\frac{5}{4} u_{h} u_{h x} u_{h x x}^{2}\left\{\frac{1}{\left(1+u_{h x}^{2}\right)^{7 / 2}}-\frac{1}{\left(1+\hat{u}_{h x}^{2}\right)^{7 / 2}}+7 \frac{\hat{u}_{h x}\left(u_{h x}-\hat{u}_{h x}\right)}{\left(1+\hat{u}_{h x}^{2}\right)^{9 / 2}}\right\} \\
& +\frac{35}{4} \frac{\hat{u}_{h x}\left(u_{h x}-\hat{u}_{h x}\right)}{\left(1+\hat{u}_{h x}^{2}\right)^{9 / 2}}\left(\hat{u}_{h} \hat{u}_{h x} \hat{u}_{h x x}^{2}-u_{h} u_{h x} u_{h x x}^{2}\right)+\frac{3}{4} \frac{\hat{u}_{h x}\left(u_{h x}-\hat{u}_{h x}\right)}{\left(1+\hat{u}_{h x}^{2}\right)^{5 / 2}}\left(\frac{\hat{u}_{h x}}{\hat{u}_{h}}-\frac{u_{h x}}{u_{h}}\right) \\
& +\frac{1}{4} \frac{u_{h x}-\hat{u}_{h x}}{\left(1+\hat{u}_{h x}^{2}\right)^{3 / 2}}\left(\frac{1}{u_{h}}-\frac{1}{\hat{u}_{h}}\right)+\frac{1}{4} \frac{u_{h x}}{u_{h}}\left\{\frac{1}{\left(1+u_{h x}^{2}\right)^{3 / 2}}-\frac{1}{\left(1+\hat{u}_{h x}^{2}\right)^{3 / 2}}+3 \frac{\hat{u}_{h x}\left(u_{h x}-\hat{u}_{h x}\right)}{\left(1+\hat{u}_{h x}^{2}\right)^{5 / 2}}\right\}, \\
& C=-\frac{1}{2} \frac{\left(\hat{u}_{h}-u_{h}\right)\left(\hat{u}_{h x x}-u_{h x x}\right)}{\left(1+\hat{u}_{h x}^{2}\right)^{5 / 2}}-\frac{5}{2} \frac{\hat{u}_{h x}\left(u_{h x}-\hat{u}_{h x}\right)\left(\hat{u}_{h} \hat{u}_{h x x}-u_{h} u_{h x x}\right)}{\left(1+\hat{u}_{h x}^{2}\right)^{7 / 2}} \\
& -\frac{1}{2} u_{h} u_{h x x}\left\{\frac{1}{\left(1+u_{h x}^{2}\right)^{5 / 2}}-\frac{1}{\left(1+\hat{u}_{h x}^{2}\right)^{5 / 2}}+5 \frac{\hat{u}_{h x}\left(u_{h x}-\hat{u}_{h x}\right)}{\left(1+\hat{u}_{h x}^{2}\right)^{7 / 2}}\right\} \text {. }
\end{aligned}
$$


It follows from the above representation that

$$
|A|+|B|+|C| \leqslant C\left(\left|\theta_{h}\right|^{2}+\left|\theta_{h x}\right|^{2}+\left|\theta_{h x x}\right|^{2}\right) \quad \text { in } \bar{I} \times\left[0, \hat{T}_{h}\right) .
$$

Inserting (5.13) into 5.6) taking into account (5.7) and 5.15) we obtain

$$
\begin{aligned}
c_{2}\left\|\theta_{h t}\right\|^{2}+\psi^{\prime}(t) & \leqslant C\left\|\theta_{h t}\right\|\left\|\theta_{h}\right\|+C\left\|\theta_{h}\right\|_{H^{2}}^{2}-\frac{\mathrm{d}}{\mathrm{d} t} \int_{I} f e_{h} \theta_{h x}+\epsilon\left\|\theta_{h t}\right\|^{2}+C_{\epsilon}\left(h^{8}+\left\|\theta_{h}\right\|_{H^{1}}^{2}\right) \\
& \leqslant \epsilon\left\|\theta_{h t}\right\|^{2}-\frac{\mathrm{d}}{\mathrm{d} t} \int_{I} f e_{h} \theta_{h x}+C_{\epsilon}\left(h^{8}+\left\|\theta_{h}\right\|_{H^{2}}^{2}\right) .
\end{aligned}
$$

If we choose $\epsilon=c_{2} / 2$ and integrate with respect to time taking into account 4.9 we deduce

$$
\begin{aligned}
\frac{c_{2}}{2} \int_{0}^{t}\left\|\theta_{h t}\right\|^{2} \mathrm{~d} s+\psi(t) \leqslant & \psi(0)+C\left(\left\|e_{h}(\cdot, t)\right\|\left\|\theta_{h x}(\cdot, t)\right\|+\left\|e_{h}(\cdot, 0)\right\|\left\|\theta_{h x}(\cdot, 0)\right\|\right) \\
& +C h^{8}+C \int_{0}^{t}\left\|\theta_{h}\right\|_{H^{2}}^{2} \mathrm{~d} s \\
\leqslant & C\left\|\theta_{h}(\cdot, t)\right\|_{H^{1}}^{2}+C h^{8}+\int_{0}^{t}\left\|\theta_{h}\right\|_{H^{2}}^{2} \mathrm{~d} s .
\end{aligned}
$$

Here we also used the fact that $\psi(0) \leqslant C\left\|\theta_{h}(\cdot, 0)\right\|_{H^{2}}^{2} \leqslant C h^{8}$, the second inequality being a consequence of 5.1. Recalling 5.14 and using the interpolation inequality $\left\|\theta_{h x}\right\|^{2} \leqslant \epsilon\left\|\theta_{h}\right\|_{H^{2}}^{2}+$ $C_{\epsilon}\left\|\theta_{h}\right\|^{2}$ we infer, with $\epsilon=\mu /(2 C)$,

$$
\frac{c_{2}}{2} \int_{0}^{t}\left\|\theta_{h t}\right\|^{2} \mathrm{~d} s+\frac{\mu}{2}\left\|\theta_{h}(\cdot, t)\right\|_{H^{2}}^{2} \leqslant C\left\|\theta_{h}(\cdot, t)\right\|^{2}+C h^{8}+C \int_{0}^{t}\left\|\theta_{h}\right\|_{H^{2}}^{2} \mathrm{~d} s .
$$

Note that in view of 5.1 ,

$$
\left\|\theta_{h}(\cdot, t)\right\|^{2} \leqslant C h^{8}+2 \int_{0}^{t}\left\|\theta_{h}\right\|\left\|\theta_{h t}\right\| \mathrm{d} s \leqslant \epsilon \int_{0}^{t}\left\|\theta_{h t}\right\|^{2} \mathrm{~d} s+C h^{8}+C_{\epsilon} \int_{0}^{t}\left\|\theta_{h}\right\|^{2} \mathrm{~d} s .
$$

Inserting this estimate into the above inequality we finally obtain, after choosing $\epsilon$ small enough,

$$
\frac{c_{2}}{4} \int_{0}^{t}\left\|\theta_{h t}\right\|^{2} \mathrm{~d} s+\frac{\mu}{2}\left\|\theta_{h}(\cdot, t)\right\|_{H^{2}}^{2} \leqslant C h^{8}+C \int_{0}^{t}\left\|\theta_{h}\right\|_{H^{2}}^{2} \mathrm{~d} s .
$$

Gronwall's inequality implies

$$
\int_{0}^{\hat{T}_{h}}\left\|\theta_{h t}\right\|^{2} \mathrm{~d} s+\sup _{0 \leqslant t \leqslant \hat{T}_{h}}\left\|\theta_{h}(\cdot, t)\right\|_{H^{2}}^{2} \leqslant C h^{8} .
$$

We can now prove that $\hat{T}_{h}=T$. If not, we would have $\hat{T}_{h}<T$. Combining 3.2 with an inverse estimate, 4.9) and 5.16) we obtain

$$
\begin{aligned}
\left\|u(\cdot, t)-u_{h}(\cdot, t)\right\|_{W^{2, \infty}} & \leqslant\left\|u(\cdot, t)-I_{h} u(\cdot, t)\right\|_{W^{2, \infty}}+\left\|I_{h} u(\cdot, t)-u_{h}(\cdot, t)\right\|_{W^{2, \infty}} \\
& \leqslant C h^{2}\|u(\cdot, t)\|_{W^{4, \infty}}+C h^{-1 / 2}\left\|I_{h} u(\cdot, t)-u_{h}(\cdot, t)\right\|_{H^{2}} \\
& \leqslant C h^{2}+C h^{-1 / 2}\left(\left\|u(\cdot, t)-I_{h} u(\cdot, t)\right\|_{H^{2}}+\left\|\rho_{h}(\cdot, t)\right\|_{H^{2}}+\left\|\theta_{h}(\cdot, t)\right\|_{H^{2}}\right) \\
& \leqslant C h^{3 / 2}, \quad 0 \leqslant t \leqslant \hat{T}_{h} .
\end{aligned}
$$


In view of (5.2) there exists $h_{0}>0$ which only depends on $c_{0}, C_{0}, \eta, T$ and the solution $u$ such that

$$
\frac{3}{4} c_{0} \leqslant u_{h}(x, t) \leqslant \frac{3}{2} C_{0}, \quad\left|u_{h x}(x, t)\right|,\left|u_{h x x}(x, t)\right| \leqslant \frac{3}{2} C_{0} \quad \forall(x, t) \in I \times\left[0, \hat{T}_{h}\right],
$$

provided that $0<h \leqslant h_{0}$. However, we could then extend the discrete solution to an interval $\left[0, \hat{T}_{h}+\delta\right]$ for some $\delta>0$ with

$$
c_{0} / 2 \leqslant u_{h}(x, t) \leqslant 2 C_{0}, \quad\left|u_{h x}(x, t)\right|,\left|u_{h x x}(x, t)\right| \leqslant 2 C_{0} \quad \forall(x, t) \in I \times\left[0, \hat{T}_{h}+\delta\right],
$$

contradicting the definition of $\hat{T}_{h}$. Thus $\hat{T}_{h}=T$. Combining (5.16) with (4.9) and 4.14 completes the proof of Theorem 3.1

\section{Numerical results}

In order to confirm the theoretically derived error estimates one needs a test problem with a known exact solution of the continuous problem. To this purpose we consider the more general problem

$$
\int_{I} \frac{u(\cdot, t) u_{t}(\cdot, t) \phi}{\sqrt{1+u_{x}(\cdot, t)^{2}}}+\left\langle\tilde{W}^{\prime}(u(\cdot, t)), \phi\right\rangle=\int_{I} f(\cdot, t) u(\cdot, t) \phi \quad \forall \phi \in H_{0}^{2}(I), t \in(0, T] .
$$

The function $f: \bar{I} \times[0, T] \rightarrow \mathbb{R}$ is computed from a given smooth function $u: \bar{I} \times[0, T] \rightarrow \mathbb{R}$ according to

$$
f(x, t)=\frac{u_{t}(x, t)}{\sqrt{1+u_{x}(x, t)^{2}}}-\left\{\Delta_{\Gamma} H+2 H\left(H^{2}-K\right)\right\},
$$

while the expressions $\Delta_{\Gamma} H$ and $H^{2}-K$ are given in terms of $u(x, t)$ by the formulae in Remark 2.1.

Then the equation (3.6) of the semidiscrete problem $3.6-3.8$ is generalized to

$$
\int_{I} \frac{u_{h}(\cdot, t) u_{h t}(\cdot, t) \phi_{h}}{\sqrt{1+u_{h x}(\cdot, t)^{2}}}+\left\langle\tilde{W}^{\prime}\left(u_{h}(\cdot, t)\right), \phi_{h}\right\rangle=\int_{I} f(\cdot, t) u_{h}(\cdot, t) \phi_{h} \quad \forall \phi_{h} \in X_{h 0}, t \in(0, T] .
$$

Now, we want to solve this semidiscrete problem numerically with the boundary conditions 3.7 and the practically more suitable initial condition

$$
u_{h}(\cdot, 0)=I_{h} u_{0}
$$

where the function $u_{0}: \bar{I} \rightarrow \mathbb{R}$, in the case of the academic test problem with a given analytical solution $u(x, t)$, is defined as $u_{0}(x):=u(x, 0)$. We will write (6.2) as a finite-dimensional nonlinear system of ODEs. The time-exact solution $u_{h}(\cdot, t) \in X_{h}$ for all $t \in[0, T]$ can be represented as

$$
u_{h}(x, t)=\sum_{j=-1}^{2 N} c_{j}(t) \varphi_{j}(x) \quad \forall x \in \bar{I},
$$

where the time dependent coefficients $c_{j}:[0, T] \rightarrow \mathbb{R}$ are unknown and the space basis functions $\varphi_{j} \in C^{1}(\bar{I})$ are defined as follows. For each element $K_{i}:=\left[x_{i-1}, x_{i}\right], i=1, \ldots, N$, we have 
$\left.\varphi_{j}\right|_{K_{i}} \in \mathbb{P}_{3}$ for all $j$. The first set of basis functions $\left\{\varphi_{2 i-1}\right\}$ is responsible for the point values of the discrete function at the nodes $x_{i}$, i.e.,

$$
\varphi_{2 i-1}\left(x_{k}\right)=\delta_{i, k}, \quad \varphi_{2 i-1}^{\prime}\left(x_{k}\right)=0 \quad \forall i, k \in\{0, \ldots, N\} .
$$

The second set $\left\{\varphi_{2 i}\right\}$ is responsible for the values of the $x$-derivatives at the nodes $x_{i}$, i.e.,

$$
\varphi_{2 i}\left(x_{k}\right)=0, \quad \varphi_{2 i}^{\prime}\left(x_{k}\right)=\delta_{i, k} \quad \forall i, k \in\{0, \ldots, N\} .
$$

These conditions for the definition of the basis functions $\varphi_{j}$ imply the following meaning of the coefficients $c_{j}(t)$ in 6.4):

$$
c_{2 i-1}(t)=u_{h}\left(x_{i}, t\right), \quad c_{2 i}(t)=u_{h x}\left(x_{i}, t\right) \quad \forall i=0, \ldots, N .
$$

The Dirichlet boundary conditions (3.7) are satisfied if and only if

$$
c_{-1}(t)=\alpha_{l}, \quad c_{0}(t)=\beta_{l}, \quad c_{2 N-1}(t)=\alpha_{r}, \quad c_{2 N}(t)=\beta_{r} \quad \forall t \in[0, T] .
$$

The modified initial condition 6.3 is implemented as

$$
u_{h}(x, 0)=u_{0, h}(x)=\sum_{j=-1}^{2 N} c_{j}^{0} \varphi_{j}(x)
$$

where $u_{0, h}:=I_{h} u_{0} \in X_{h}$, i.e.,

$$
c_{2 i-1}^{0}:=u_{0}\left(x_{i}\right), \quad c_{2 i}^{0}:=\frac{\mathrm{d} u_{0}}{\mathrm{~d} x}\left(x_{i}\right) \quad \forall i=0, \ldots, N .
$$

Therefore, the initial conditions for the unknown coefficient functions $c_{j}(t)$ are $c_{j}(0)=c_{j}^{0}$. To get the equations for the remaining unknown functions $c_{j}(\cdot), j=1, \ldots, 2 N-2$, we choose in 6.2 the test functions $\phi_{h}=\varphi_{i} \in X_{h, 0}$ with $i=1, \ldots, 2 N-2$. Then the semidiscrete problem 6.2), (3.7), 6.3 is equivalent to the following nonlinear system of ODEs of dimension $d:=2 N-2$ for the vector function $U:[0, T] \rightarrow \mathbb{R}^{d}$ of the coefficient functions with $U_{j}(t):=c_{j}(t)$ :

$$
\begin{aligned}
M(U(t)) U^{\prime}(t) & =F(t, U(t)) \quad \forall t \in(0, T], \\
U(0) & =U^{0},
\end{aligned}
$$

where the initial vector is $U^{0}=\left(c_{j}^{0}\right) \in \mathbb{R}^{d}$. The matrix-valued function $M: \mathbb{R}^{d} \rightarrow \mathbb{R}^{d \times d}$ is given by

$$
M(U)=\left(M(U)_{i, j}\right) \quad \text { with } \quad M(U)_{i, j}:=\int_{I} \frac{u_{h}(U) \varphi_{j} \varphi_{i}}{\sqrt{1+u_{h x}(U)^{2}}} .
$$

Here, $u_{h}(U) \in X_{h}$ denotes the function

$$
u_{h}(U)(x)=\sum_{j=-1}^{2 N} U_{j} \varphi_{j}(x) \quad \forall x \in I
$$

with the convention that, for a given vector $U \in \mathbb{R}^{d}$, we formally define $U_{-1}=\alpha_{l}, U_{0}=\beta_{l}$, 
$U_{2 N-1}=\alpha_{r}$ and $U_{2 N}=\beta_{r}$. The function $F:[0, T] \times \mathbb{R}^{d} \rightarrow \mathbb{R}^{d}$ is defined by its components $F_{i}(t, U)$ as

$$
F_{i}(t, U):=\int_{I} f(\cdot, t) u_{h}(U) \varphi_{i}-\left\langle\tilde{W}^{\prime}\left(u_{h}(U)\right), \varphi_{i}\right\rangle .
$$

Under the assumption that $u_{h}(U) \geqslant c_{1}$ on $\bar{I}$, the nonlinear mass matrix $M(U)$ is symmetric and positive definite such that its inverse is well-defined. So the problem 6.6 is equivalent to the ODE system in the classical form

$$
\begin{aligned}
U^{\prime}(t) & =\tilde{F}(t, U(t)):=M(U(t))^{-1} F(t, U(t)) \quad \forall t \in(0, T], \\
U(0) & =U^{0} .
\end{aligned}
$$

In order to solve this ODE system numerically we compute approximations $U^{n} \approx U\left(t_{n}\right)$ of the exact solution $U:[0, T] \rightarrow \mathbb{R}^{d}$ at the discrete time levels $t_{n}:=n \tau, n=0, \ldots, M$, where $\tau:=$ $T / M$ is the time step size corresponding to a given number $M$ of equidistant time intervals. Due to the stiffness of the ODE system we should apply an A-stable time discretization scheme. A first candidate would be the implicit Euler scheme, but this would require, due to its first order accuracy, a very small time step to guarantee that the time discretization error is of the size of the spatial error $\mathcal{O}\left(h^{4}\right)$. Therefore, we apply the A-stable Crank-Nicolson scheme which is of second order accuracy. A comparison of different time discretizations for the Willmore flow problem will be an object of future work. We compute the approximations $U^{n}$ of the Crank-Nicolson scheme successively, i.e., from a known vector $U^{n-1}$, we compute the next vector $U^{n}$ as the solution of the nonlinear system of equations

$$
U^{n}=U^{n-1}+\frac{\tau}{2}\left\{\tilde{F}\left(t_{n-1}, U^{n-1}\right)+\tilde{F}\left(t_{n}, U^{n}\right)\right\} .
$$

This system is solved by means of a quasi-Newton method applied to the homogeneous defect equation

$$
\tilde{d}(U):=U-U^{n-1}-\frac{\tau}{2}\left\{\tilde{F}\left(t_{n-1}, U^{n-1}\right)+\tilde{F}\left(t_{n}, U\right)\right\}=0_{\mathbb{R}^{d}} .
$$

Instead of solving with the correct Jacobi matrix $J=D_{U} \tilde{d}\left(U^{\text {old }}\right)$ we solve with the quasi-Jacobi matrix

$$
\tilde{J}:=I+\frac{\tau}{2}\left(M^{\text {old }}\right)^{-1}\left\{\gamma_{s} S-D_{U} F\left(t_{n}, U^{\text {old }}\right)\right\}
$$

where $M^{\text {old }}:=M\left(U^{\text {old }}\right), \gamma_{s} \geqslant 0$ is a relatively small parameter and $S$ a stabilizing matrix defined by

$$
S=\left(S_{i, j}\right) \quad \text { with } \quad S_{i, j}:=\int_{I} \varphi_{j}^{\prime \prime} \varphi_{i}^{\prime \prime} .
$$

The matrix $S$, which is symmetric and positive definite, has been introduced in order to achieve more robust convergence of the quasi-Newton iteration. Numerical experiments have shown that in some cases a positive value of $\gamma_{s}$ indeed yields better convergence compared to the choice $\gamma_{s}=0$. We compute the new Newton iterate as $U^{\text {new }}:=U^{\text {old }}-V^{\text {new }}$ where we get the correction $V^{\text {new }}$ by solving the linear system

$$
\tilde{J} V^{\text {new }}=\tilde{d}\left(U^{\text {old }}\right),
$$

which is practically done by solving the equivalent system

$$
A V^{\text {new }}=M^{\text {old }}\left(U^{\text {old }}-U^{n-1}-\frac{\tau}{2} \tilde{F}\left(t_{n-1}, U^{n-1}\right)\right)-\frac{\tau}{2} F\left(t_{n}, U^{\text {old }}\right)
$$


with

$$
A:=M^{\text {old }}+\frac{\tau}{2}\left\{\gamma_{s} S-D_{U} F\left(t_{n}, U^{\text {old }}\right)\right\} .
$$

For a sufficiently small time step size $\tau$, the convergence of this quasi-Newton iteration is relatively fast. In the computations below, the damping factor of the norm of the defect in one Newton step was between 0.05 in the first steps and 0.4 in the last steps during one nonlinear solve. The number of Newton iterations per time step to achieve that the norm of the defect is less than $10^{-14}$ was at most 17.

An analysis of the fully discrete scheme is beyond the scope of this paper. Since the CrankNicolson scheme is of order $\tau^{2}$, we expect an error bound of the form

$$
\left\|e_{h, \tau}\right\|_{i}:=\max _{0 \leqslant n \leqslant M}\left\|u\left(\cdot, t_{n}\right)-u_{h, \tau}\left(\cdot, t_{n}\right)\right\|_{H^{i}} \leqslant C\left(\tau^{2}+h^{4}\right) h^{-i}, \quad i=0,1 .
$$

We want to confirm this estimate by a numerical example where we prescribe the exact solution as

$$
u(x, t):=\frac{3}{2}+\cos (\pi t)\left\{\cos (x)-c+s x^{2}\right\}
$$

with $s=\sin (1) / 2, c=\cos (1)+s$ and compute the corresponding function $f(x, t)$ as explained above. This solution satisfies the Dirichlet boundary conditions with $\alpha_{l}=\alpha_{r}=3 / 2$ and $\beta_{l}=$ $\beta_{r}=0$. For the length $T$ of the time interval we take $T=1$. The space grid is chosen equidistant with the mesh size $h$. We consider a series of four computations where in the $k$-th calculation the discretization parameters $h_{k}$ and $\tau_{k}$ are chosen such that

$$
h_{k}:=2^{-k}, \quad \tau_{k}:=\frac{h_{k}^{2}}{64}=\frac{1}{64 \cdot 4^{k}}, \quad k=1, \ldots, 4 .
$$

If the error estimate $(6.12)$ is correct we should see an error reduction like

$$
\left\|e_{h, \tau}\right\|_{i} \leqslant C h_{k}^{4-i} \leqslant C\left(\frac{2^{i}}{16}\right)^{k},
$$

i.e., if we double the number of elements and take four times more time steps as in the previous calculation then the error $\left\|e_{h, \tau}\right\|_{0}$ should be reduced by a factor of $1 / 16$ and the error $\left\|e_{h, \tau}\right\|_{1}$ by a factor of $1 / 8$. Table 1 shows the results of the corresponding four calculations and confirms the theoretically predicted behavior. The error norms have been computed by means of the 4point Gaussian quadrature formula applied to the space integrals such that the quadrature error is negligibly small. For the parameter $\gamma_{s}$ in the Crank-Nicolson scheme, we have used the value 0.5.

TABLE 1

Error norms $\left\|u-u_{h, \tau}\right\|_{i}$ for the test problem with known analytical solution and experimental orders of convergence (EOC).

\begin{tabular}{|r|r||||c|c||c|c|}
\hline$h$ & $\tau$ & $\left\|u-u_{h, \tau}\right\|_{0}$ & EOC & $\left\|u-u_{h, \tau}\right\|_{1}$ & EOC \\
\hline $1 / 2$ & $1 / 256$ & $1.245 e-4$ & & $8.691 e-4$ & \\
$1 / 4$ & $1 / 1024$ & $7.774 e-6$ & 4.001 & $1.084 e-4$ & 3.003 \\
$1 / 8$ & $1 / 4096$ & $4.860 e-7$ & 4.000 & $1.355 e-5$ & 3.001 \\
$1 / 16$ & $1 / 16384$ & $3.038 e-8$ & 4.000 & $1.693 e-6$ & 3.000 \\
\hline
\end{tabular}


As a second numerical test example we consider the Willmore flow problem $(2.12)-(2.14)$ and its discretization (6.2), (3.7), 6.3) with $f(x, t) \equiv 0$. For the Dirichlet boundary data, we choose $\alpha_{l}=\alpha_{r}=0.2$ and $\beta_{l}=\beta_{r}=0$. We have used $T=1$ as the length of the time interval. As initial condition $u_{0}$ we used an analytically motivated $C^{1}$ function that satisfies the boundary conditions (see [6]). To investigate the convergence behavior we performed a series of five computations where in the $k$-th calculation we used a boundary adapted grid consisting of $N E L(k):=3 \cdot 2^{k-1}$ elements with mesh-size $h_{k}=(12 / 5) 2^{-k}$. In order to neglect the error of the time discretization by the Crank-Nicolson scheme, we have chosen the relatively fine time step size $\tau_{k}=1 /\left(25 \cdot 4^{k}\right)$ in the $k$-th calculation. As an indicator for the accuracy of the computed time discrete solution we have presented in the last column of Table 2 the distance of the actual $k$-th solution to the solution with time step size $\tau_{k} / 2$. As an approximation for the exact solution we computed a reference solution $\tilde{u}(x, t)$ on a mesh with mesh-size $h=3 / 80$ constructed by uniform refinement from the 5 -th mesh. We used a uniform time step size of $\tau=1 / 204800 \approx 4.883 e-6$. For the stabilization parameter in the Newton iteration of the Crank-Nicolson scheme, the value $\gamma_{s}=0$ provided the best convergence rates in all five computations. Figure 1 shows, for the coarsest mesh with $N E L(1)=3$ elements, the discrete solution $u_{h_{1}, \tau_{1}}(\cdot, T)$ at the final time $T$ and the reference solution $\tilde{u}(\cdot, T)$. One can see the high accuracy of the $C^{1}$ finite elements even on this very coarse grid. For this coarse space

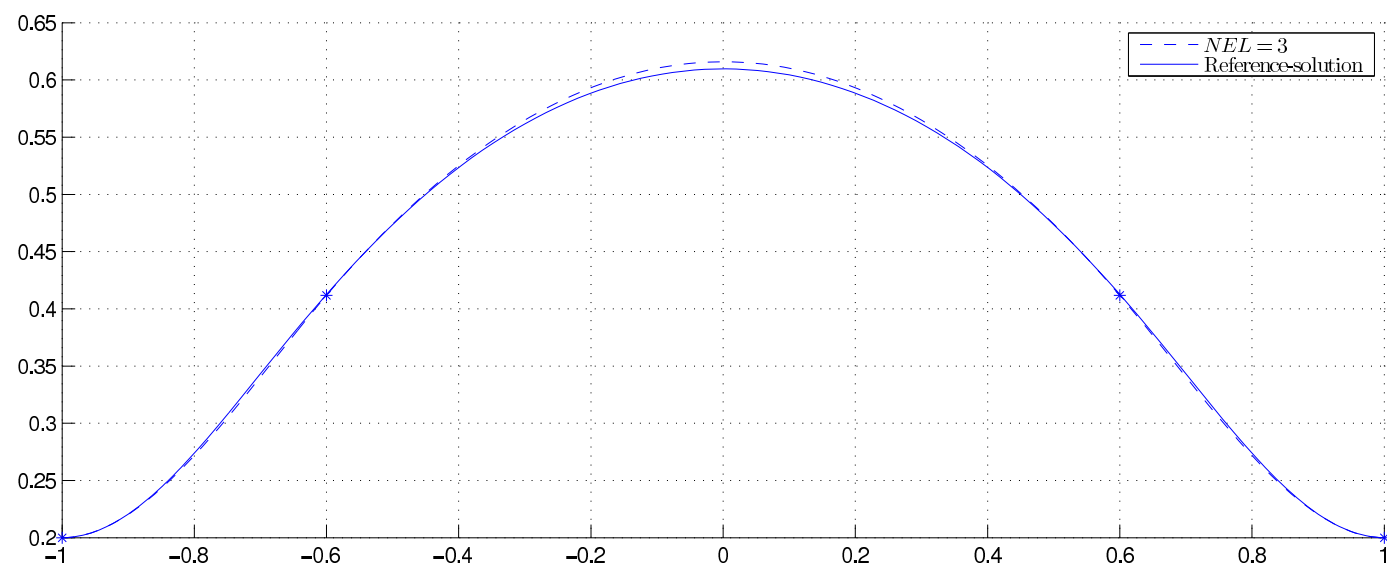

FIG. 1. Reference solution (solid line) and approximate semidiscrete solution (dashed line) at final time $T=1$ on a mesh with three elements indicated by the ' $*$ '.

grid, Figure 2 shows a plot of the discrete Willmore energy over the time. Table 2 shows how the $L^{2}$-error of the approximate semidiscrete solution at the final time $T=1$ is reduced if the mesh from the previous calculation is uniformly refined by bisecting each element. The error norms have been computed by means of the 4-point Gaussian quadrature formula applied on each element of the grid for the reference solution. For $h \leqslant 3 / 40$ one seems to be in the asymptotic regime of $\mathcal{O}\left(h^{4}\right)$ convergence.

Finally, we present in Figure 3 the graph of the stationary discrete solution for the non-symmetric boundary data $\alpha_{l}=0.5, \alpha_{r}=0.3, \beta_{l}=-2$ and $\beta_{r}=-4$ determined by computing the Willmore flow up to time $T=0.5$. 


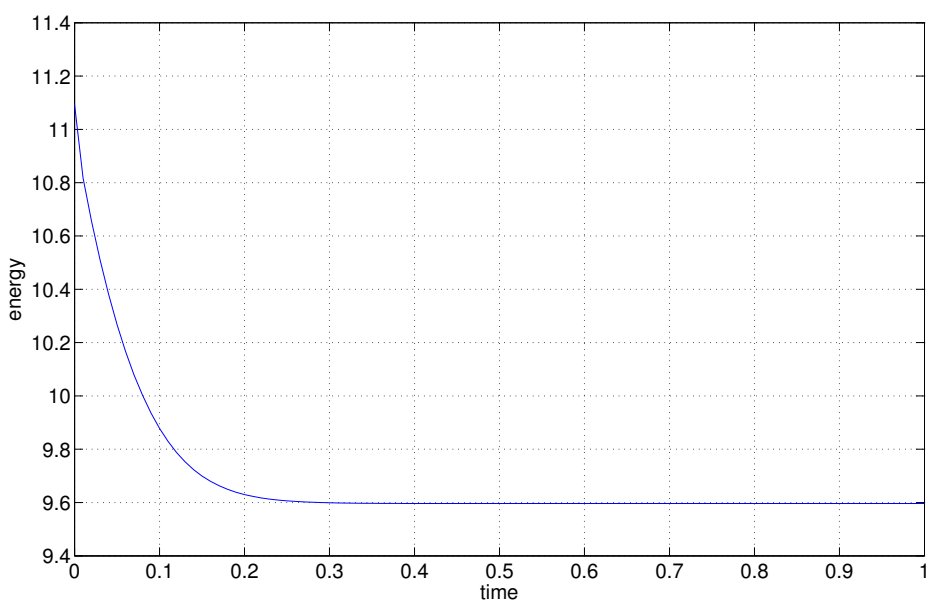

FIG. 2. Discrete Willmore energy over the time computed on the space grid from Figure 1 with three elements $(h=6 / 5)$ using a time step size of $\tau=1 / 100$

\section{TABLE 2}

$L^{2}$-error between the reference solution $\tilde{u}(\cdot, T)$ and the approximate semidiscrete solution $u_{h, \tau}(\cdot, T)$ computed with time step size $\tau$ at final time $T=1$ on a grid with mesh size $h$ and experimental orders of convergence (EOC).

\begin{tabular}{|r|r||c|c|c|c|}
\hline$h$ & $\tau$ & $h^{4}$ & $\left\|u_{h, \tau}(T)-\tilde{u}(T)\right\|_{0}$ & EOC & $\left\|u_{h, \tau}(T)-u_{h, \tau / 2}(T)\right\|_{0}$ \\
\hline $6 / 5$ & $1 / 100$ & $2.074 e+0$ & $1.426 e-3$ & & $1.090 e-8$ \\
$3 / 5$ & $1 / 400$ & $1.296 e-1$ & $5.141 e-4$ & 1.472 & $8.329 e-8$ \\
$3 / 10$ & $1 / 1600$ & $8.100 e-3$ & $1.469 e-4$ & 1.807 & $2.804 e-6$ \\
$3 / 20$ & $1 / 6400$ & $5.062 e-4$ & $1.574 e-5$ & 3.222 & $1.348 e-6$ \\
$3 / 40$ & $1 / 25600$ & $3.164 e-5$ & $1.060 e-6$ & 3.892 & $1.214 e-7$ \\
\hline
\end{tabular}

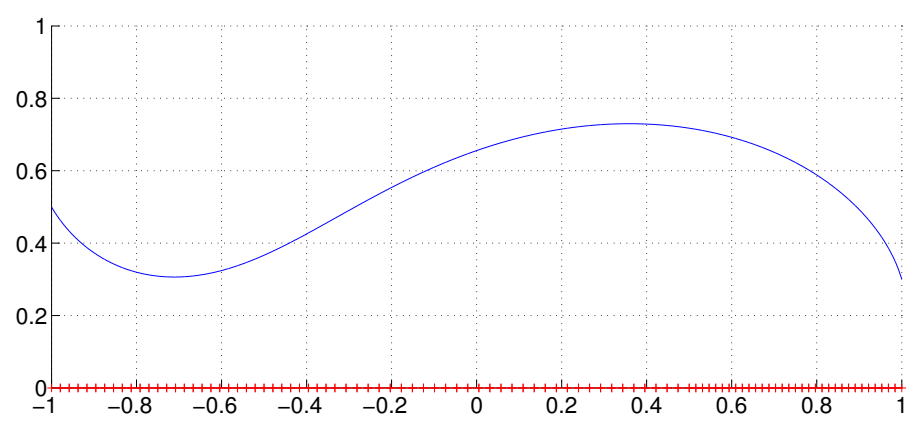

FIG. 3. Stationary discrete solution for nonsymmetric boundary data. 


\section{Acknowledgements}

The authors gratefully acknowledge financial support from the German Research Council (DFG) through grant DE 611/5-1.

\section{REFERENCES}

1. Barrett, J. W., Garcke, H., \& NÜrnberg, R. Parametric approximation of Willmore flow and related geometric evolution equations. SIAM J. Sci. Comput. 31 (2008), 225-253. Zbl 1186.65133 MR 2460777

2. Bonito, A., Nochetto, R. H., \& Pauletti, M. S. Parametric FEM for geometric biomembranes. J. Comput. Phys. 229 (2010), 3171-3188. Zbl pre05693254 MR 2601095

3. BRAKKe, K. A. The surface evolver. Experiment. Math. 1 (1992), 141-165. Zbl 0769.49033 MR 1203871

4. Clarenz, U., Diewald, U., Dziuk, G., Rumpf, M., \& Rusu, R. A finite element method for surface restoration with smooth boundary conditions. Computer Aided Geom. Design 21 (2004), 427-445. Zbl 1069.65546 MR 2058390

5. Dall' Acqua, A., Deckelnick, K., \& Grunau, H.-CH. Classical solutions to the Dirichlet problem for Willmore surfaces of revolution. Adv. Calc. Var. 1 (2008), 379-397. Zbl 1194.49060 MR 2480063

6. Dall' Acqua, A., Fröhlich, S., Grunau, H.-Ch., \& Schieweck, F. Symmetric Willmore surfaces of revolution satisfying arbitrary Dirichlet boundary data. Adv. Calc. Var, to appear.

7. Deckelnick, K., \& DZIUK, G. Error analysis of a finite element method for the Willmore flow of graphs. Interfaces Free Bound. 8 (2006), 21-46. Zbl 1102.35047 MR 2231251

8. Deckelnick, K., \& Grunau, H.-Ch. A Navier boundary value problem for Willmore surfaces of revolution. Analysis 29 (2009), 229-258. Zbl 1188.53065 MR 2568881

9. Droske, M., \& Rumpf, M. A level set formulation for Willmore flow. Interfaces Free Bound. 6 (2004), 361-378. Zbl 1062.35028 MR 2095338

10. DZIUK, G. Computational parametric Willmore flow. Numer. Math. 111 (2008), 55-80. Zbl 1158.65073 MR 2448203

11. Eidelman, S. D., \& Zhitarashu, N. V. Parabolic Boundary Value Problems. Birkhäuser, Basel (1998). Zbl 0893.35001 MR 1632789

12. HelfRICH, W. Elastic properties of lipid bilayers: Theory and possible experiments. Z. Naturforsch. 28c (1973), 693-703.

13. HSU, L., Kusner, R., \& Sullivan, J. Minimizing the squared mean curvature integral for surfaces in space forms. Experiment. Math. 1 (1992), 191-207. Zbl 0778.53001 MR 1203874

14. Kuwert, E., \& Schätzle, R. The Willmore flow with small initial energy. J. Differential Geom. 57 (2001), 409-441. Zbl 1035.53092 MR 1882663

15. KUWERT, E., \& SCHÄtZLE, R. Removability of point singularities of Willmore surfaces. Ann. of Math. 160 (2004), 315-357. Zbl 1078.53007 MR 2119722

16. Mayer, U. F., \& Simonett, G. A numerical scheme for axisymmetric solutions of curvature-driven free boundary problems, with applications to the Willmore flow. Interfaces Free Bound. 4 (2002), 89-109. Zbl 1005.65095 MR 1877537

17. Olischläger, N., \& RUMPF, M. Two step time discretization of Willmore flow. In: Mathematics of Surfaces, XIII (York, 2009), Lecture Notes in Comput. Sci. 5654, Springer (2009), 278-292. Zbl pre05622622

18. RUSU, R. An algorithm for the elastic flow of surfaces. Interfaces Free Bound. 7 (2005), 229-239. Zbl pre02215421 MR 2171130 
19. Schätzle, R. The Willmore boundary value problem. Calc. Var. Partial Differential Equations 37 (2010), 275-302. Zbl 1188.53006 MR 2592972

20. Seifert, U. Configurations of fluid membranes and vesicles. Adv. Phys. 46 (1997), 13-137.

21. WheELER, M. F. A priori $L_{2}$ error estimates for Galerkin approximations to parabolic partial differential equations. SIAM J. Numer. Anal. 10 (1973), 723-759. MR 0351124

22. Willmore, T. J. Riemannian Geometry. Oxford Univ. Press, New York (1993). Zbl 0797.53002 MR 1261641 